\title{
THE EFFECT OF POLARIZATION AND InGaN QUANTUM WELL SHAPE IN MULTIPLE QUANTUM WELL LIGHT EMITTING DIODE HETEROSTRUCTURES
}

\author{
A Thesis \\ Presented to \\ The Faculty of the Department of Materials Engineering \\ California Polytechnic State University \\ San Luis Obispo \\ In Partial Fulfillment of the Requirements of the Degree \\ Master of Science and Engineering \\ With Specialization in Materials Engineering
}

By

Patrick Miroslav McBride

June 2012 
(C)2012

Patrick Miroslav McBride

ALL RIGHTS RESERVED

ii | Page 


\section{COMMITTEE MEMBERSHIP}

TITLE:

AUTHOR:

DATE SUBMITTED:

COMMITTEE CHAIR:

COMMITTEE MEMBER:

COMMITTEE MEMBER:

COMMITTEE MEMBER:
THE EFFECT OF POLARIZATION AND InGaN QUANTUM WELL SHAPE IN MULTIPLE QUANTUM WELL LIGHT EMITTING DIODE HETEROSTRUCTURES

Patrick Miroslav McBride

June 2012

Dr. Richard Savage

Dr. Linda Vanasupa

Dr. Thomas Gutierrez

Dr. Robert Echols 


\section{Abstract \\ THE EFFECT OF POLARIZATION AND InGaN QUANTUM WELL SHAPE IN MULTIPLE QUANTUM WELL LIGHT EMITTING DIODE HETEROSTRUCTURES}

by Patrick McBride

Previous research in InGaN/GaN light emitting diodes (LEDs) employing semi-classical drift-diffusion models has used reduced polarization constants without much physical explanantion. This paper investigates possible physical explanations for this effective polarization reduction in InGaN LEDs through the use of the simulation software SiLENSe. One major problem of current LED simulations is the assumption of perfectly discrete transitions between the quantum well (QW) and blocking layers when experiments have shown this to not be the case. The In concentration profile within InGaN multiple quantum well (MQW) devices shows much smoother and delayed transitions indicative of indium diffusion and drift during common atomic deposition techniques (e.g. molecular beam epitaxy, chemical vapor deposition). In this case the InGaN square $\mathrm{QW}$ approximation may not be valid in modeling the devices' true electronic behavior. A simulation of a $3 Q W$ InGaN/GaN LED heterostructure with an AIGaN electron blocking layer is discussed in this paper. Polarization coefficients were reduced to $70 \%$ and $40 \%$ empirical values to simulate polarization shielding effects. QW shapes of square (3 $\mathrm{nm}$ ), trapezoidal, and triangular profiles were used to simulate realistic $\mathrm{QW}$ shapes. The $\mathrm{J}-\mathrm{V}$ characteristic and electron-hole wavefunctions of each device were monitored. Polarization reduction decreased the onset voltage from $4.0 \mathrm{~V}$ to $3.0 \mathrm{~V}$ while $\mathrm{QW}$ size reduction decreased the onset voltage from $4.0 \mathrm{~V}$ to $3.5 \mathrm{~V}$. The increased current density in both cases can be attributed to increased wavefunction overlap in the QWs.

Keywords: Gallium Nitride, Indium Nitride, Semiconductor, Polarization, Quantum Mechanics, Drift Diffusion, Carrier Recombination, Quantum Well, Heterostructure, Doping, Electrodynamics, Maxwell's Equations, Light Absorption, Perturbation Theory 


\section{Acknowledgements}

I would first like to thank my advisor, Dr. Richard Savage, for sticking with me for the past couple years. Without him, I would never have discovered UCSB and been able to do the wonderful research that I have done. I also would never have met Paul Bonderson, who has graciously helped me fund my master's project and my Ph.D. program at UCSB through the Bonderson fellowship.

I would also like to thank Dr. Chris Van de Walle and, soon to be doctor, Qimin Yan, both from the Materials Department at UCSB, for their wonderful insight and help. I would also like to thank everyone else in the Van de Walle group for their help and exciting group meetings every week.

I also want to thank the professors in the Cal Poly Physics Department, particularly Dr. Thomas Gutierrez and Dr. Robert Echols, for all their support these past few years and all the inspiration they gave me to dive into quantum mechanics and start down the path of condensed matter physics.

I would also like to thank my wonderful girlfriend Sarah, who sat with me and helped me edit my thesis the whole way.

I also owe a big thanks to all my friends who have kept me sane these past years: Ravi, Alex, Jono, Taylor, Eli, Annie, Sarah, David, Hackman, Martin, Logan, Clay, Kari, and of course Keith for showing me why I wanted to be a physicist.

Lastly, I would like to thank my mom and dad for helping me in school the whole way through and inspiring me to be like the academics that they are. And my brother for making all these years just a bit more interesting and being mom and dad's biologist like I never could. 


\section{Table of Contents}

List of Figures vii

List of Tables ix

1 Introduction 1

1.1 History of Semiconductors . . . . . . . . . . . . . . . . . 2

1.2 Modern LEDs: Race to the blue LED . . . . . . . . . . . . . . . . 4

1.2.1 Polarization in GaN LEDs . . . . . . . . . . . . . . . 6

1.3 Problem Statement . . . . . . . . . . . . . . . . . . . . . . 7

1.4 Semiconductor Physics . . . . . . . . . . . . . . . . 8

1.4.1 Classical Electrodynamics . . . . . . . . . . . . . . . . . 9

1.4.2 Quantum Mechanics in Semiconductors . . . . . . . . . 13

1.4.3 Semi-Classical Drift-Diffusion Model . . . . . . . . . . . . 24

2 Methods and Materials $\quad 32$

2.1 Device Design . . . . . . . . . . . . . . . . . . . . . . . . . . . . 32

2.1.1 Internal Structure . . . . . . . . . . . . . . . . . . 33

2.2 Measurement Parameters . . . . . . . . . . . . . . . . . . 34

3 Results $\quad 35$

3.1 Effect of Polarization Reduction . . . . . . . . . . . . . . . . . 35

3.1.1 Band Diagram with Reduced Polarization . . . . . . . . . 35

3.1.2 Wavefunctions with Reduced Polarization . . . . . . . . . 37

3.2 Effect of Quantum Well Shape . . . . . . . . . . . . . . . . . . 38

3.2.1 Band Diagram with Altered Quantum Well Shape . . . . . 39

3.2.2 Wavefunctions with Altered Quantum Well Shape . . . . . 41

4 Discussion $\quad 42$

4.1 Analysis of Polarization Reduction . . . . . . . . . . . . . . . . . 42

4.2 Analysis of Quantum Well Shape . . . . . . . . . . . . . . . . . 43

4.3 Polarization vs. Well Shape . . . . . . . . . . . . . . . . . . . . . 44

5 Conclusion $\quad 45$ 


\section{List of Figures}

1 The luminous efficacy can be shown throughout the years since the 1940 s for various lighting motifs. Here we see a recent upheaval of SSL efficacy surpassing incandescent and even that of CFL and HID. ${ }^{1} \ldots$. . . . . . . . . . . . . . . . . . . . .

2 The current and projected site electricity consumption is shown up to 2030. This shows that current market trends in LED adoption will lead to a $46 \%$ savings by $2030{ }^{2} \ldots$. . . . . . . . . . . .

3 Figure depicts the rapid changes in GaN SSL technology through a graph of $\eta_{\text {ext }}$ from $1970-2000 .^{3}$ It is important to note that the log scale on the vertical axis implies exponential growth in the latter half of the 1990s and presumably until now based on Figure 1 as well.

The wurtzite crystal structure of GaN. Due to the higher electronegativity of nitrogen, a dipole moment forms that points away from nitrogen and towards gallium. Because the bond length along the c-direction is longer than the other bond directions, a spontaneous polarization is formed.

5 Diagram depicting $A$ ) the absorption and $B$ ) the emission of light in a two state system predicted by Fermi's golden rule (Equation 47) . . . . . . . . . . . . . . . . . . . Depiction of the interband radiative transition with $(A)$ the generation of an electron-hole pair and $(B)$ the recombination of an electron-hole pair.

Depiction of SRH non-radiative transitions with $(A)$ electron capture, (B) electron emission, (C) hole capture, and (D) hole emission. . . . . . . . . . . . . . . . . . . . .

8 Depiction of Auger non-radiative trap state transitions with (A) electron capture with electron excitation (left) and hole excitation (right), (B) electron emission with excited electron (left) and excited hole (right), (C) hole capture with hole excitation (left) and electron excitation (right), and (D) hole emission with excited hole (left) and excited electron (right).

9 Depiction of Auger non-radiative band to band state transitions showing $(A)$ electron capture (left) and emission (right) with an excited electron, and (B) electron capture (left) and emission (right) with an excited hole.

10 Picture of the layer structure for the InGaN/GaN MQW heterostructure employed in SiLENSe simulations.

11 Graphical representation of indium concentration across one QW for the $3 \mathrm{~nm}$ (flat), $2 \mathrm{~nm}, 1 \mathrm{~nm}$, and $0 \mathrm{~nm}$ (triangular) devices. These are to be used as simple and qualitative representations of a non square QW as shown in Kisielowski et al. ${ }^{4}$. . . . . . . . .

12 The current density vs. bias voltage of the $\ln _{0.2} \mathrm{Ga}_{0.8} \mathrm{~N} 3 \mathrm{QW}$ heterostructure for polarization coefficients at 100\% (blue), $70 \%$ (green), and $40 \%$ (red) of their empirical values. 
13 The band diagrams of the $\ln _{0.2} \mathrm{Ga}_{0.8} \mathrm{~N} 3 \mathrm{QW}$ heterostructure at a $3.5 \mathrm{~V}$ bias for polarization coefficients at $100 \%, 70 \%$, and $40 \%$ of their empirical values. Reducing polarization causes band flattening in the QW and barrier regions. This increases the electron-hole overlap and subsequently increases current density across the device. . . . . . . . . . . . . . . . . .

14 The electron-hole wavefunctions for the middle $\mathrm{QW}$ in the $\mathrm{In}_{0.2} \mathrm{Ga}_{0.8} \mathrm{~N} 3 \mathrm{QW}$ heterostructure at a $3.5 \mathrm{~V}$ bias for polarization coefficients at $(A) 100 \%$ (overlap $=10.4 \%$ ), (B) $70 \%$ (overlap = $25.0 \%$ ), and (C) $40 \%$ (overlap $=52.7 \%$ ) of their empirical values. (D) This figure shows how the electron-hole wavefunctions tend toward each other as polarization is reduced (inset graphically shows the trend with overlap). This increases the electron-hole overlap, which is in accordance with the increased current density calculated.

15 The current density vs. bias voltage of the $\ln _{0.2} \mathrm{Ga}_{0.8} \mathrm{~N} 3 \mathrm{QW}$ heterostructure for trapezoidal indium profiles with base lengths of $3 \mathrm{~nm}$ (blue), $1 \mathrm{~nm}$ (green), and $0 \mathrm{~nm}$ (red). . . . . . . . . . .

16 The band diagrams of the $\ln _{0.2} \mathrm{Ga}_{0.8} \mathrm{~N} 3 \mathrm{QW}$ heterostructure at a $3.5 \mathrm{~V}$ bias for trapezoidal indium profiles with base lengths of 3 $\mathrm{nm}$ (square), $1 \mathrm{~nm}$, and $0 \mathrm{~nm}$ (triangular). Narrowing the flat indium composition in the QW region reduces the polarization induced band bending, which thereby increases electron-hole overlap while raising the GaN barrier energy. The former leads to an increase in current density while the latter leads to a decrease. This creates similar, but not identical, effects to polarization reduction. . . . . . . . . . . . . . . . . . . .

17 The electron-hole wavefunctions for the middle $\mathrm{QW}$ in the $\mathrm{In}_{0.2} \mathrm{Ga}_{0.8} \mathrm{~N} 3 \mathrm{QW}$ heterostructure at a $3.5 \mathrm{~V}$ bias for trapezoidal indium profiles with base lengths of (A) $3 \mathrm{~nm}$ (overlap $=10.4 \%$ ), (B) $1 \mathrm{~nm}$ (overlap $=26.6 \%$ ), and (C) $0 \mathrm{~nm}$ (overlap $=32.9 \%$ ). (D) This figure shows how the electron and hole wavefunctions move closer together as the flat indium region is reduced (inset graphically shows the trend with overlap). A definite increase in the electron energy is seen as overlap increases, creating a blueshift in the energy spectrum. . . . . . . . . . . 


\section{List of Tables}

I Polarization Constants for Wurtzite Group III Nitrides ${ }^{5}$. . . . . . 12 


\section{Introduction}

For those unfamiliar with the current stages of lighting technology, light emitting diodes (LEDs) have recently come to the forefront of energy efficiency and light output technologies. According to the solid state lighting (SSL) of the Department of Energy (DOE), current commercial LEDs have even surpassed the luminous efficacy of compact fluorescent lights (CFLs) and high intensity discharge lamps (HIDs) (Figure 1). In addition to efficiency, LEDs also boast a longer lifetime as well as more versatility due to their more compact nature. ${ }^{2}$

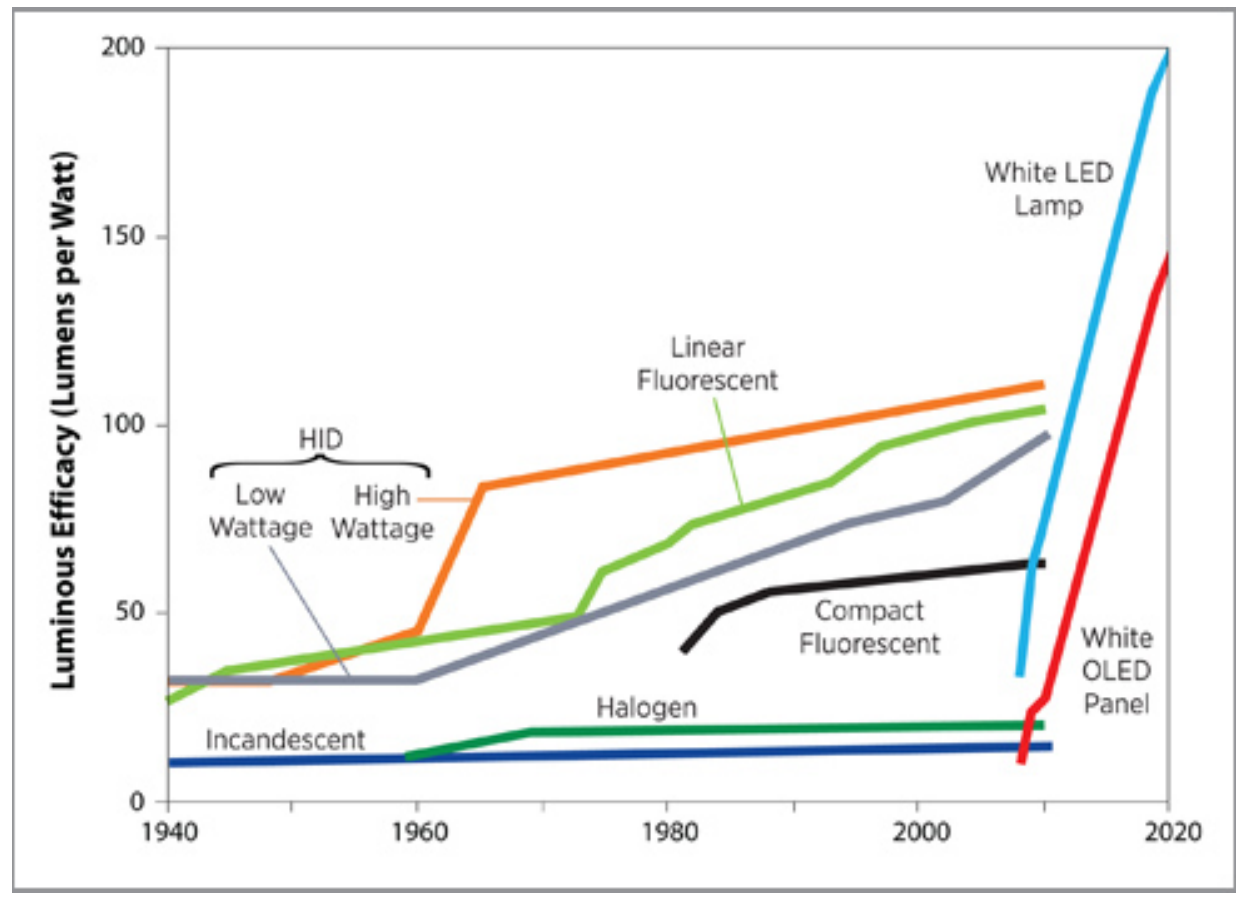

Figure 1: The luminous efficacy can be shown throughout the years since the 1940 s for various lighting motifs. Here we see a recent upheaval of SSL efficacy surpassing incandescent and even that of CFL and HID. ${ }^{1}$

The U.S. currently estimates its site electricity consumption to be over 3,500 terawatt-hours (TWh), $18 \%$ of which is occupied by lighting technologies. This accounts for roughly 694 TWh, or 7.9 quadrillion Btu (quads), of energy being used to light only the United States in 2010. ${ }^{2}$ At current energy prices, this amounts to roughly $\$ 70$ billion being spent on lighting in the U.S. alone. By 
current DOE estimates (Figure 2), if the market demand increases the use of LEDs to $74 \%$ of the current market lumen-hours by 2030 , it will save the nation a total of $\$ 30$ billion. Hence, the recent push for improvement in LED lighting efficiency has important consequences for energy savings and efficiency.

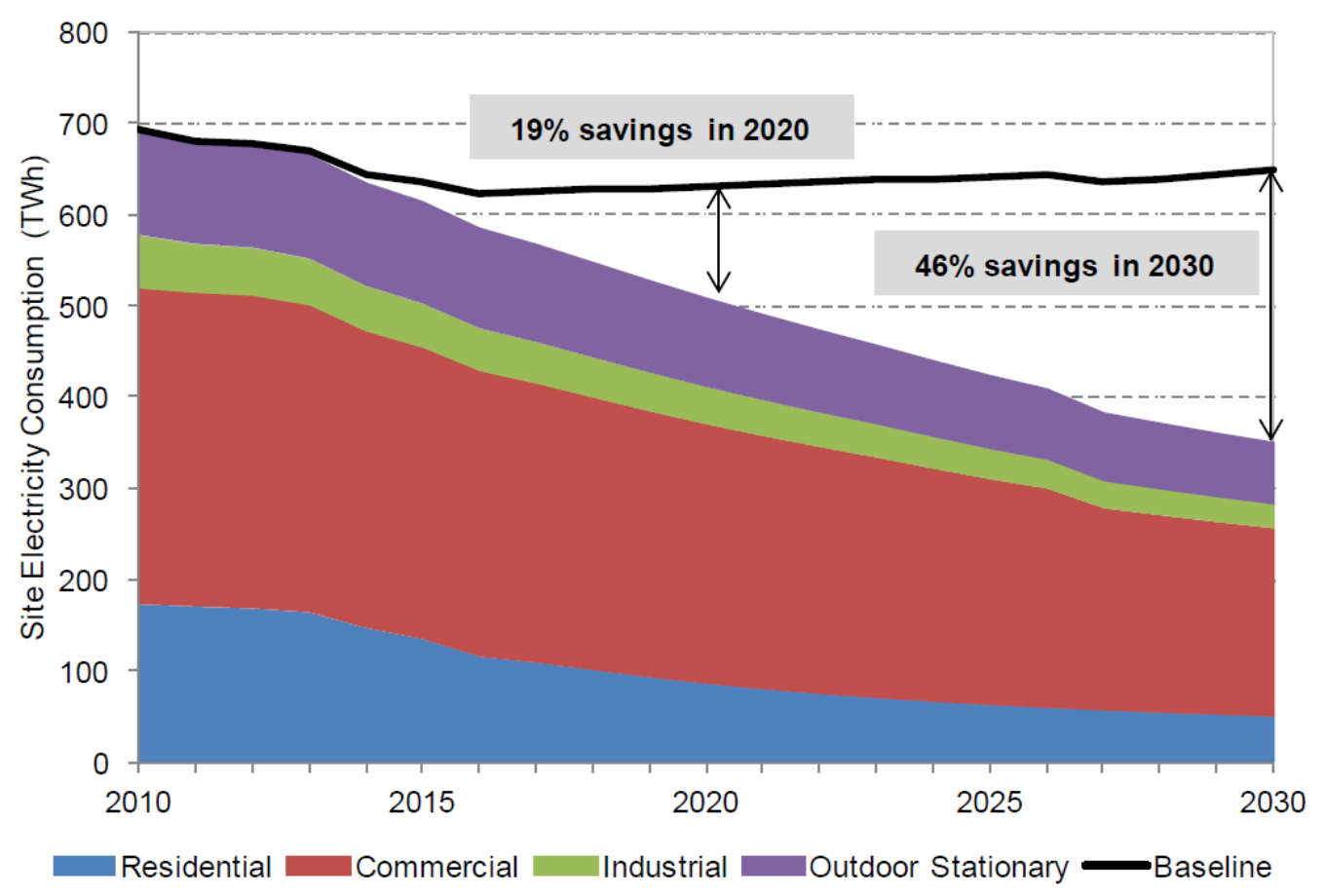

Figure 2: The current and projected site electricity consumption is shown up to 2030. This shows that current market trends in LED adoption will lead to a $46 \%$ savings by $2030 .^{2}$

\subsection{History of Semiconductors}

Light emitting diodes (LEDs) have been a staple of the lighting industry for the past decade or so, but many of the current accomplishments have come from a rich past of semiconductor devices as well as the struggles that have been overcome in the process of their creation. Although the bulk of this thesis is based on LED devices, specifically the group III-V nitride semiconductors, a background in semiconductor device technology and the role of theory in conjunction with experiment is crucial in obtaining a full appreciation for how solid state theory has driven much of the research in semiconductor 
electronics. A quick look at the history of LEDs shows a 20 year gap around 1930-1950 in which LED research was driven to a standstill due to a lack of theoretical understanding. ${ }^{6}$ In order to prevent such recurrences and drive further technological innovations in the semiconductor industry, maintaining and developing a strong theoretical basis and applying these theoretical trends to experimental data is paramount.

Much of the early research in semiconductor LEDs can be attributed to Oleg V. Losev, ${ }^{6,7}$ a Russian physicist with a knack for creative experimental techniques and observation. However, the first recorded discovery of the LED was by H.J. Round in 1907 during his research on SiC crystal detectors for use in the recent phenomenon of wireless radio transmission. ${ }^{6}$ Yet further research into this phenomenon was not pursued by researchers at the time, including Round, because they were preoccupied with the development of radio wave detector technology. No headway was seen with LEDs until 1922 when Losev first detected light emission from a silicon carbide point crystal detector. Moreover, most LED research in the 1920s was soley through the efforts of Losev under the guise of rectifier research. By 1930, Losev had developed theories similar to those now regarding $\mathrm{p}-\mathrm{n}$ junctions and majority carrier injection, but his theory was incomplete for he did not yet know of holes as a charge carrier type. Losev's discoveries were phenomenal, yet the world was not quite ready for his research.

Since materials for semiconductors were expensive to manufacture and perfect crystals were difficult to obtain, research in this field was slow and lacked theory for guidance. The quantum theory had just gotten underway with Schrödinger's discovery of the electronic wave equation in 1926. Felix Bloch made the earliest headway into solid state quantum theory when he developed a theory of electronic solids in $1928 .^{8}$ In his theory, Bloch developed the idea of a periodic electronic wavefunction to satisfy the Schrödinger equation with the periodic potential found in crystalline solids. This allowed for further 
developments by Rudolf Perierls in 1929 that began the concept of holes, or "defect electrons" as they were once called, in crystalline solids. ${ }^{6}$ The idea of holes helped to explain what many physicists up to this point could not: the positive Hall effect in metals and semiconductors. The positive Hall effect shows that certain materials (e.g., beryllium) have positive charge carriers, which was against the commonly held belief that electrons were the charge carriers. This understanding of charge carriers in solids provides the theoretical basis for arguably the most important discovery in semiconductor device electronics: the p-n junction.

The physical basis of the p-n junction was first described in the 1949 Bell System Technical Journal paper ${ }^{9}$ by William Shockley, which set the stage for all field effect devices and, of course, LEDs. Creating the theory of light emission from semiconductor diodes only took a few years, and its creation allowed Kurt Lehovec to combine the idea of electroluminescence and diode theory into a coherent theory of LEDs. ${ }^{10}$ Within a few months Lehovec's paper, published in 1952, led to the creation of a p-n junction LED device and hence was born the modern LED.

\subsection{Modern LEDs: Race to the blue LED}

The first LEDs produced in the 1950s were typically gallium arsenide based and only emitted in the infrared. As technology began to improve, researchers began attempting to produce LEDs that would emit in all areas of the optical spectrum. However, as LEDs achieved color ranges from red to green, the only blue LEDs known were silicon carbide and gallium nitride. These two compounds are problematic because, until the latter part of the century, they both had notoriously poor efficiencies.

Silicon carbide was the first blue LED discovered in the 1920s, but it was not refined until the $1960 \mathrm{~s} .{ }^{11}$ However, the electrical to optical conversion 
efficiencies, or external quantum efficiency

$$
\eta_{\text {ext }} \equiv \frac{\text { photons extracted }}{\text { charge carriers injected }}
$$

of these early SiC LEDs were extremely poor at a low $0.005 \%$. Even by the early 1990s, when the first commercial SiC LEDs hit the market, their efficiency never got higher than $0.03 \%$. This poor efficiency resulted from SiC's indirect band transition, which led researchers to look for a direct band gap material in the blue end of the spectrum.

The production of gallium based LEDs, beginning with the GaAs infrared emitting LED that was discovered in the 1960s, paralleled the development of SiC LEDs. ${ }^{11}$ This eventually proved profitable with the creation of GaP, and in 1969, Maruska et al. discovered a blue direct transition, GaN. ${ }^{12}$ Unfortunately, it was very difficult to grow perfect single crystals of $\mathrm{GaN}$ at the time, which made p-type GaN almost impossible to make. Research began to wane until 1992 when Shuji Nakamura developed a thermal annealing method for Mg-doped GaN. ${ }^{13}$ This immediately made GaN blue LEDs a strong contender in the commercial sector with a $\eta_{e x t}$ of around $1.5 \%$. The efficiency of GaN LEDs has been increasing ever since as shown in Figure $3 .^{3}$

The commercially viable blue GaN LED now made possible the production of white light LEDs, thereby revolutionizing the lighting industry. Since white is really just the colors red, green, and blue mixed in certain proportions, it would seem that putting together a red, green, and blue LED would do the trick. While this is true, commercial white LEDs actually use only one blue LED and shine light through a down-converting phosphor (typically $\mathrm{Y}_{3} \mathrm{Al}_{5} \mathrm{O}_{12} \cdot \mathrm{Ce}^{3+}$ ) that converts the blue light to light across the visible spectrum. Hence, a stronger understanding of GaN LEDs would directly impact every facet of the lighting industry. 


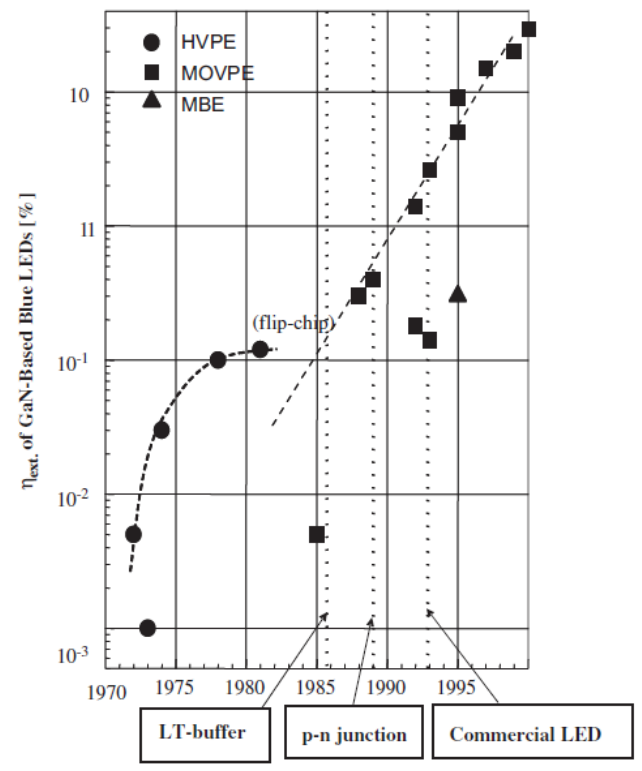

Figure 3: Figure depicts the rapid changes in GaN SSL technology through a graph of $\eta_{\text {ext }}$ from 1970-2000. ${ }^{3}$ It is important to note that the log scale on the vertical axis implies exponential growth in the latter half of the 1990s and presumably until now based on Figure 1 as well.

\subsubsection{Polarization in GaN LEDs}

Gallium nitride LEDs are typically grown on (0001) sapphire or (0001) SiC substrates, which prefer growth along the polar c-axis of the wurtzite crystal structure. ${ }^{5}$ This induces a spontaneous polarization, $\mathbf{P}_{s p}$, along the c-axis that varies for different nitrides (e.g., InN or AIN) as explained in section 1.4.

When multiple nitride materials of varying elemental compositions are placed together in a layered fashion (e.g., GaN/InN/GaN), the individual dipole moments no longer cancel along the c-axis within the material. Furthermore, due to lattice mismatch, internal strain within the nitride crystal induces a piezoelectric polarization within each section of the material. These induced polarizations form regions of positive/negative alternating sheet charge, ${ }^{5}$ which creates a capacitive effect that distorts the electric field along the c-axis and leads to band bending, ${ }^{5}$ slanted band diagrams, ${ }^{14}$ and quantum confined Stark effect. ${ }^{5,14}$ The band bending and altered band diagrams reduces the electron-hole spatial overlap in LED devices, thereby reducing the quantum 


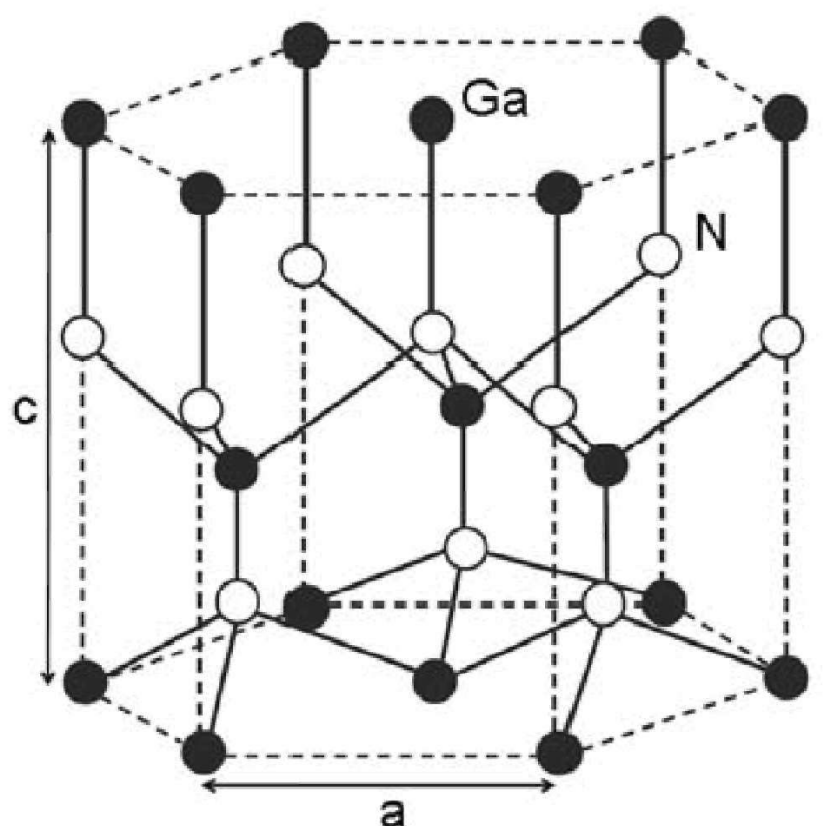

Figure 4: The wurtzite crystal structure of GaN. Due to the higher electronegativity of nitrogen, a dipole moment forms that points away from nitrogen and towards gallium. Because the bond length along the c-direction is longer than the other bond directions, a spontaneous polarization is formed.

efficiency. Furthermore, the quantum confined Stark effect tends to alter output by blueshifting the energy spectrum at higher currents. ${ }^{14}$

\subsection{Problem Statement}

Common growth techniques in group III-V GaN heterostructure LEDs tend to develop a spontaneous and piezoelectric polarization along the growth axis. This induced polarization then forms capacitive regions of alternating polarity, which tend to distort the intrinsic band structure of the GaN device. Current physical simulations of InGaN/GaN quantum well (QW) and multiple $Q W$ (MQW) LEDs that employ a quantum corrected drift-diffusion model do not lead to fully accurate experimental predictions. In some cases, a reduction in polarization constants was necessary to comply with experimental results. ${ }^{15}$ This has been justified by introducing charge shielding due to defects ${ }^{16,17}$ to effectively reduce the intrinsic polarization. ${ }^{18,19}$ The goal of this thesis is to 
determine the root cause for these "polarization shielding" effects so that we may either exploit or disprove any such mechanism in an overall goal to improve GaN-based LEDs.

\subsection{Semiconductor Physics}

The entirety of this research is based upon the physical model employed within the SiLENSe modeling package provided by STR, Inc. This system uses a semi-classical 1D drift-diffusion model of carrier transport to determine the current through an LED device given the bias voltage and certain constraints. Some of these constraints include: piezoelectric and spontaneous polarization, radiative and non-radiative recombination rates, and threading dislocation densities. ${ }^{20}$ In order for the reader to understand the research that is discussed within this paper, it is important to first go over the basic equations for what makes SiLENSe work. Therefore, the next few sections will provide as brief as possible an introduction to the basics of electrodynamics in semiconductors with an emphasis on certain concepts needed for understanding the research contained in this thesis (i.e., polarization and drift-diffusion). I will begin with classical electromagnetism in free space before generalizing these concepts to real materials (i.e., semiconductors). I then introduce some basic quantum mechanics and use this as the theoretical basis for the phenomenological drift-diffusion model of electron transport in semiconductor devices. This will provide sufficient background to extend this to heterostructure LED devices and allow the reader to understand a basic premise for how SiLENSe works. 


\subsubsection{Classical Electrodynamics}

\section{Maxwell's Equations}

The fundamental set of equations that governs all of electromagnetics was first discovered by James Clerk Maxwell in 1873 and are aptly dubbed Maxwell's equations. These equations are the fundamental basis for all of classical, and modern, electrodynamics. For a stationary charge density, $\rho$, and a moving current, $\mathbf{J} \equiv \rho \mathbf{v}$, where $\mathbf{v}$ is velocity, Maxwell's equations can be written in differential form as ${ }^{21,22}$

$$
\begin{aligned}
\nabla \cdot \mathbf{E} & =\frac{\rho}{\epsilon_{o}} & & \text { Gauss's law } \\
\nabla \times \mathbf{E} & =-\frac{\partial \mathbf{B}}{\partial t} & & \text { Faraday's law } \\
\nabla \cdot \mathbf{B} & =0 & & \text { Gauss's law } \\
\nabla \times \mathbf{B} & =\mu_{o} \mathbf{J}+\mu_{o} \epsilon_{o} \frac{\partial \mathbf{E}}{\partial t} & & \text { Ampère's law }
\end{aligned}
$$

where $\mathbf{E}$ is the electric field vector and $\mathbf{B}$ is the magnetic field vector. Taking the divergence of Ampére's law (Equation 4) gives the continuity equation:

$$
\nabla \cdot \mathbf{J}+\frac{\partial \rho}{\partial t}=0 \quad \text { Continuity Equation }
$$

Equations 1 through 4 are Maxwell's equations as they stand in a vacuum, but SiLENSe works with electromagnetic fields in a semiconductor device. Therefore, these equations must be modified to account for how the fields respond to matter. This can be done by allowing the "space" that the field exists in to exhibit tiny dipole moments, $\mathbf{p}$, that are formed by the intrinsic nature of the material in question, which gives a polarization vector, $\mathbf{P} \equiv \mathbf{p} / V$, where $V$ is volume. When no external electric fields are present, this inherent phenomenon of materials is called spontaneous polarization, and in 
semiconductors, it is a property of the atomic symmetry inherent to a material's crystal structure. This is important in GaN because the wurtzite crystal structure (Figure 4) has a different $\mathrm{Ga}-\mathrm{N}$ bond length in the c-direction preventing the complete cancellation of $\mathrm{Ga}-\mathrm{N}$ dipole moments and inducing a spontaneous polarization, $\mathbf{P}_{s p}$.

In order to find how $\mathbf{P}_{\mathrm{sp}}$ affects Maxwell's equations in matter, we can write down the potential, $\Phi(\mathbf{r})$, at a point $\mathbf{r}$ away from an arbitrary distribution of dipoles as ${ }^{21}$

$$
\Phi(\mathbf{r})=\frac{1}{4 \pi \epsilon_{o}} \int_{V^{\prime}} \frac{\mathbf{P} \cdot \hat{\mathbf{r}}}{\left|\mathbf{r}-\mathbf{r}^{\prime}\right|} d V^{\prime}
$$

Using integration by parts and the divergence theorem, this can be put into a more illuminating form:

$$
\begin{aligned}
\Phi(\mathbf{r}) & =\frac{1}{4 \pi \epsilon_{o}}\left[\int_{V^{\prime}} \frac{-\nabla^{\prime} \cdot \mathbf{P}}{\left|\mathbf{r}-\mathbf{r}^{\prime}\right|} d V^{\prime}+\oint_{A^{\prime}} \frac{\mathbf{P} \cdot \hat{\mathbf{n}}}{\left|\mathbf{r}-\mathbf{r}^{\prime}\right|} d A\right] \\
& =\frac{1}{4 \pi \epsilon_{o}}\left[\int_{V^{\prime}} \frac{\rho_{b}}{\left|\mathbf{r}-\mathbf{r}^{\prime}\right|} d V^{\prime}+\oint_{A^{\prime}} \frac{\sigma_{\mathbf{b}}}{\left|\mathbf{r}-\mathbf{r}^{\prime}\right|} d A\right]
\end{aligned}
$$

where $\rho_{b}$ and $\sigma_{b}$ are the bound volume and surface charge densities, respectively. Using the bound charge density and the free charge density, $\rho_{f}$, we can rewrite Gauss's law as

$$
\begin{aligned}
\nabla \cdot \mathbf{E} & =\frac{1}{\epsilon_{o}}\left(\rho_{f}+\rho_{b}\right) \\
\epsilon_{o} \nabla \cdot \mathbf{E} & =\rho_{f}-\nabla \cdot \mathbf{P} \\
\nabla \cdot\left(\epsilon_{o} \mathbf{E}+\mathbf{P}\right) & =\rho_{f}
\end{aligned}
$$

defining the electric displacement vector, $\mathbf{D} \equiv \epsilon_{o} \mathbf{E}+\mathbf{P}$, Gauss's law in matter becomes:

$$
\nabla \cdot \mathbf{D}=\rho_{f}
$$


A similar treatment can be done involving the magnetic field where we define the magnetic field intensity, $\mathbf{H} \equiv \frac{1}{\mu_{o}} \mathbf{B}-\mathbf{M}$, with $\mathbf{M}$ being the magnetization vector (i.e., the magnetic analogue to the polarization vector $\mathrm{P}$ ). We then obtain Maxwell's equations for electromagnetic fields in matter: ${ }^{21,22}$

$$
\begin{aligned}
\nabla \cdot \mathbf{D} & =\rho_{f} & & \text { Gauss's law } \\
\nabla \times \mathbf{E} & =-\frac{\partial \mathbf{B}}{\partial t} & & \text { Faraday's law } \\
\nabla \cdot \mathbf{B} & =0 & & \text { Gauss's law } \\
\nabla \times \mathbf{H} & =\mathbf{J}_{f}+\frac{\partial \mathbf{D}}{\partial t} & & \text { Ampère's law }
\end{aligned}
$$

\section{Spontaneous and Piezoelectric Polarization}

As shown in the previous section (Equation 7), the polarization of a material can affect the electric potential, and thereby alter the carrier energy via the Schrödinger equation (discussed in section 1.4.2). This can be seen in Poisson's equation for electrostatics:

$$
\nabla^{2} \Phi=-\frac{\rho}{\epsilon_{o}}
$$

which can be modified to include the effects of polarization

$$
-\epsilon_{o} \nabla^{2} \Phi=\rho_{f}-\nabla \cdot \mathbf{P}
$$

This equation helps to show how the curvature of electron energy band diagrams results from both free charge density and bound charge density (i.e., change in polarization across a boundary).

Spontaneous polarization in group-III nitride based devices is an intrinsic property of the crystal symmetry and has been experimentally determined for GaN, InN, and AIN homogeneous crystals ${ }^{5}$ (Table I). Using Vegard's law, 5,16 
the material parameters for an arbitrary epitaxial layer of $\ln _{x} \mathrm{Al}_{y} \mathrm{Ga}_{1-x-y} \mathrm{~N}$ can be determined from those of GaN, InN, and AIN by the following equation:

$$
\xi_{L}=x \cdot \xi_{I n N}+y \cdot \xi_{A l N}+(1-x-y) \cdot \xi_{G a N}
$$

where $\xi_{G a N}, \xi_{I n N}$, and $\xi_{A l N}$ are arbitrary material parameters (e.g., polarization constant, lattice constant) for each of the respective materials and $\xi_{L}$ is the material parameter for the alloy layer. From Equation 15, the material properties for any composition of gallium, indium, or aluminum nitride can be determined.

\begin{tabular}{cccc} 
Table I: Polarization Constants for Wurtzite Group III Nitrides & \\
\hline \hline Polarization Coefficients $\left(\mathrm{C} / \mathrm{m}^{2}\right)$ & GaN & $\operatorname{lnN}$ & AIN \\
\hline$e_{33}$ & 0.73 & 0.73 & 1.55 \\
$e_{31}$ & -0.49 & -0.49 & -0.58 \\
$e_{15}$ & -0.40 & -0.40 & -0.48 \\
$P_{s p}$ & -0.029 & -0.032 & -0.081 \\
\hline \hline
\end{tabular}

In this study it is assumed that layer's lattice constant, $a_{L}$, conforms to the GaN buffer lattice constant, $a_{G a N}$. For layers grown along the c-plane direction, this amounts to only two strain components that are equal due to the hexagonal symmetry:

$$
\epsilon_{x x}=\epsilon_{y y}=\frac{a_{G a N}-a_{L}}{a_{L}}
$$

From Equation 16 we may now compute the components of the piezoelectric polarization, $\mathbf{P}_{p z}$. By taking into account the crystal symmetry of wurtzite group-III nitrides, there are only three independent piezoelectric tensor components, $e_{i j}$, giving ${ }^{5}$

$$
\mathbf{P}_{p z}=\left(\begin{array}{c}
e_{15} \epsilon_{x z} \\
e_{15} \epsilon_{y z} \\
e_{31}\left(\epsilon_{x x}+\epsilon_{y y}\right)+e_{33} \epsilon_{z z}
\end{array}\right)
$$


which can be further simplified by noting that the only remaining strains are in the $x x$ and $y y$ directions:

$$
\mathbf{P}_{p z}=\left(\begin{array}{c}
0 \\
0 \\
e_{31}\left(\epsilon_{x x}+\epsilon_{y y}\right)
\end{array}\right)
$$

The total polarization can then be represented as

$$
\begin{aligned}
\mathbf{P}_{t o t}=P_{t o t} \hat{\mathbf{z}} & =\left[P_{s p}+P_{p z}\right] \hat{\mathbf{z}} \\
& =\left[P_{s p}+e_{31}\left(\epsilon_{x x}+\epsilon_{y y}\right)\right] \hat{\mathbf{z}} \\
& =\left[P_{s p}+2 e_{31}\left(\frac{a_{G a N}-a_{L}}{a_{L}}\right)\right] \hat{\mathbf{z}}
\end{aligned}
$$

which, in general, does not remain constant at the boundary between two crystals. Hence, at the transition between the GaN buffer and the epitaxial growth layer there is a discontinuity in $\mathbf{P}$, giving a bound surface charge density,

$$
\sigma_{b}=\hat{\mathbf{n}} \cdot\left(\mathbf{P}_{G a N}-\mathbf{P}_{L}\right)=-\hat{\mathbf{n}} \cdot \Delta \mathbf{P}
$$

It is important to note that for layered structures such as $G a N / / n G a N / G a N$, this surface charge will alternate sign, giving rise to capacitive effects.

\subsubsection{Quantum Mechanics in Semiconductors}

\section{The Schrödinger Equation}

Any discussion of quantum mechanics must always begin with the famous Schrödinger equation:

$$
\hat{H} \Psi(\mathbf{r}, t)=i \hbar \frac{\partial}{\partial t} \Psi(\mathbf{r}, t)
$$


where $\Psi(\mathbf{r}, t)$ is the particle's wavefunction, $\hbar$ is the reduced Planck constant (defined by $\hbar \equiv h / 2 \pi \approx 1.05 \times 10^{-34} \mathrm{~J} \cdot \mathbf{s}$ ), $i$ is the imaginary number (defined by $i \equiv \sqrt{-1}$ ), and $\hat{H}$ is the Hamiltonian operator given by

$$
\begin{aligned}
\hat{H} & =\left[-\frac{p^{2}}{2 m}+U(\mathbf{r}, t)\right] \\
& =\left[-\frac{\hbar^{2}}{2 m} \nabla^{2}+U(\mathbf{r}, t)\right]
\end{aligned}
$$

where $\hat{\mathbf{p}} \equiv-i \hbar \nabla$ is the canonical momentum operator, $m$ is the particle's mass, and $U(\mathbf{r}, t)$ is its potential energy. The Schrödinger equation (Equation 21) is a second order partial differential equation that, in principle, allows one to calculate the probability density, $|\Psi(\mathbf{r}, t)|^{2}$, of any particle when the Hamiltonian of the system is known.

We can use separation of variables to write $\Psi(\mathbf{r}, t)=\psi(\mathbf{r}) \phi(t)$, where $\psi(\mathbf{r})$ is the spatial part of the wavefunction and $\phi(t)$ is the time part. The Schrödinger equation can then be rewritten as two equations,

$$
\begin{aligned}
\hat{H} \psi & =E \psi \\
i \hbar \dot{\phi} & =E \phi
\end{aligned}
$$

where $E$ is the energy of the particle and $\dot{\phi}$ is shorthand for $\frac{d \phi}{d t}$. Equation 23 is known as the time-independent Schrödinger equation and cannot be solved until a potential energy function is given. However, Equation 24 can readily be solved to give

$$
\phi=e^{-i E t / \hbar}
$$

where $e$ is the base to the natural logarithm. The general solution to the time-dependent Schrödinger equation (Equation 21) is then of the form

$$
\Psi(\mathbf{r}, t)=\sum_{n} c_{n} \psi_{n} e^{-i E_{n} t / \hbar}
$$


where the sum is over all available states of the system and $c_{n}$ is a normalization coefficient such that the integral of $|\Psi|^{2}$ over all space is unity. Using Dirac notation, this can be expressed as

$$
\langle\Psi \mid \Psi\rangle \equiv \int_{-\infty}^{\infty} \Psi^{*} \Psi \mathrm{d} V=1
$$

where $\Psi^{*}$ is the complex conjugate of $\Psi$. We can further expand Equation 26 at time $t=0$ to obtain more information about the $c_{n}$ coefficients. Using the orthonormality condition $\left\langle\psi_{m} \mid \psi_{n}\right\rangle=\delta_{m n}$ where $\delta_{m n}$ is the Kronecker delta function, we obtain

$$
\begin{aligned}
\langle\Psi \mid \Psi\rangle & =\sum_{m} c_{m}^{*}\left\langle\psi_{m}\left|\sum_{n} c_{n}\right| \psi_{n}\right\rangle \\
& =\sum_{m} \sum_{n} c_{m}^{*} c_{n}\left\langle\psi_{m} \mid \psi_{n}\right\rangle \\
& =\sum_{m, n} c_{m}^{*} c_{n} \delta_{m n} \\
& =\sum_{m}\left|c_{m}\right|^{2}
\end{aligned}
$$

where $\left|c_{m}\right|^{2}$ is interpreted as the probability of finding the particle in state $m$ with energy $E_{m}$. It then follows from this and Equations 26 and 27 that

$$
\sum_{n}\left|c_{n}\right|^{2}=1
$$

\section{Periodic Potentials}

All crystalline materials have a periodic lattice structure; therefore, an electron will be influenced by a periodic potential energy function. A periodic potential has the form:

$$
U(\mathbf{r})=U(\mathbf{r}+\mathbf{R})
$$


where $\mathbf{R}=n_{1} \mathbf{a}_{1}+n_{2} \mathbf{a}_{2}+n_{3} \mathbf{a}_{3}$ with $\mathbf{a}_{1}, \mathbf{a}_{2}$, and $\mathbf{a}_{3}$ being lattice basis vectors and $n_{1}, n_{2}$, and $n_{3}$ being integers. The time-independent Schrödinger equation can then be written

$$
\hat{H} \psi(\mathbf{r})=E(\mathbf{k}) \psi(\mathbf{r})
$$

where the energy $E(\mathbf{k})$ is now a function of the electron's wave vector $\mathbf{k}$. The Bloch theorem states that the general solution to Equation 29 is then

$$
\psi_{n \mathbf{k}}(\mathbf{r})=e^{i \mathbf{k} \cdot \mathbf{r}} u_{n \mathbf{k}}(\mathbf{r})
$$

where $\psi_{n \mathbf{k}}$ is the wavefunction for the $n^{\text {th }}$ band along the direction $\mathbf{k}$ with energy $E_{n}(\mathbf{k})$ and $u_{n \mathbf{k}}$ is a periodic function satisfied by

$$
u_{n \mathbf{k}}(\mathbf{r})=u_{n \mathbf{k}}(\mathbf{r}+\mathbf{R})
$$

Plugging Equation 30 into Equation 29 and writing it in terms of $u_{n \mathbf{k}}$ gives

$$
\left[\frac{p^{2}}{2 m}+U(\mathbf{r})+\frac{\hbar}{m} \mathbf{k} \cdot \hat{\mathbf{p}}\right] u_{n \mathbf{k}}=\left[E_{n}(\mathbf{k})-\frac{\hbar^{2} k^{2}}{2 m}\right] u_{n \mathbf{k}}
$$

If we look for extremal solutions near $\mathbf{k}=0$, then the $E_{n}(\mathbf{k})$ are approximately quadratic in $\mathrm{k}$ and the electron dispersion relation can be expressed ${ }^{22}$

$$
E_{c}(\mathbf{k})=E_{c}(0)+\frac{\hbar^{2} k^{2}}{2 m_{e}^{*}}
$$

where $E_{c}(\mathbf{k})$ is the conduction band energy and $m_{e}^{*}$ is the effective mass of the electron, which is typically a tensor quantity. The quantity $E_{c}(0)$ is typically taken to be measured from the top of the valence band where $E_{v}(0)=0$, making the conduction band energy $E_{c}(0)=E_{g}$ where $E_{g} \equiv E_{c}-E_{v}$ is the band gap energy of the material. When holes are present, a similar dispersion 
relation can be written: ${ }^{22}$

$$
E_{v}(\mathbf{k})=E_{v}(0)-\frac{\hbar^{2} k^{2}}{2 m_{h}^{*}}
$$

where $m_{h}^{*}$ is the effective mass of the holes and is typically a tensor quantity.

\section{Time-Dependent Perturbations}

For a two level system in a superposition of states $\Psi_{a}$ and $\Psi_{b}$, we have the general wavefunction,

$$
\Psi(\mathbf{r}, t)=c_{a} \psi_{a} e^{-i E_{a} t / \hbar}+c_{b} \psi_{b} e^{-i E_{b} t / \hbar}
$$

where $\left|c_{a}\right|^{2}=1$ and $\left|c_{b}\right|^{2}=0$ are the probability of finding the system at $E_{a}$ and $E_{b}$, respectively, at time $t=0$. The functions $\psi_{a}$ and $\psi_{b}$ are solutions to the time-independent Hamiltonian, $\hat{H}^{0}$, before any perturbation. We can then introduce the perturbing Hamiltonian $\hat{H}(t)=\hat{H}^{0}+\hat{H}^{\prime}(t)$ and demand that solutions to

$$
\hat{H} \Psi=i \hbar \dot{\Psi}
$$

are written as a linear combination of the original $\Psi_{a}$ and $\Psi_{b}$ such that ${ }^{23}$

$$
\Psi=c_{a}(t) \psi_{a} e^{-i E_{a} t / \hbar}+c_{b}(t) \psi_{b} e^{-i E_{b} t / \hbar}
$$

where the coefficients $c_{i}$ and $c_{f}$ are now functions of time. Applying the $\hat{H}$ operator to $\Psi$, we obtain

$$
\left(\hat{H}^{0}+\hat{H}^{\prime}\right)\left(c_{a} \psi_{a} e^{-i E_{a} t / \hbar}+c_{b} \psi_{b} e^{-i E_{b} t / \hbar}\right)=i \hbar \frac{\partial}{\partial t}\left(c_{a} \psi_{a} e^{-i E_{a} t / \hbar}+c_{b} \psi_{b} e^{-i E_{b} t / \hbar}\right)
$$


which is disorderly, but by using the chain rule on the right hand side and employing $\hat{H}^{0} \Psi=i \hbar \dot{\Psi}$, this equation can be simplified to

$$
c_{a} \hat{H}^{\prime} \psi_{a} e^{-i E_{a} t / \hbar}+c_{b} \hat{H}^{\prime} \psi_{b} e^{-i E_{b} t / \hbar}=i \hbar\left(\dot{c}_{a} \psi_{a} e^{-i E_{a} t / \hbar}+\dot{c}_{b} \psi_{b} e^{-i E_{b} t / \hbar}\right)
$$

In order to isolate $\dot{c}_{a}$, we multiply both sides by $\left\langle\psi_{a}\right|$ and exploit orthonormality to obtain

$$
c_{a} H_{a a}^{\prime} e^{-i E_{a} t / \hbar}+c_{b} H_{a b}^{\prime} e^{-i E_{b} t / \hbar}=i \hbar \dot{c}_{a} e^{-i E_{a} t / \hbar}
$$

and then we solve for $\dot{c}_{a}$,

$$
\dot{c}_{a}=-\frac{i}{\hbar}\left[c_{a} H_{a a}^{\prime}+c_{b} H_{a b}^{\prime} e^{-i\left(E_{b}-E_{a}\right) t / \hbar}\right]
$$

where the matrix element notation $H_{i j}^{\prime}=\left\langle\psi_{i}\left|\hat{H}^{\prime}\right| \psi_{j}\right\rangle$ is used. Similarly, for $\dot{c}_{b}$ we obtain

$$
\dot{c}_{b}=-\frac{i}{\hbar}\left[c_{b} H_{b b}^{\prime}+c_{a} H_{b a}^{\prime} e^{i\left(E_{b}-E_{a}\right) t / \hbar}\right]
$$

If we assume that the perturbing $\hat{H}^{\prime}$ is linear in space, an approximation that can generally be made, then the diagonal elements vanish and $H_{a a}^{\prime}=H_{b b}^{\prime}=0$, which gives

$$
\begin{aligned}
\dot{c}_{a} & =-\frac{i}{\hbar} c_{b} H_{a b}^{\prime} e^{-i\left(E_{b}-E_{a}\right) t / \hbar} \\
\dot{c}_{b} & =-\frac{i}{\hbar} c_{a} H_{b a}^{\prime} e^{i\left(E_{b}-E_{a}\right) t / \hbar}
\end{aligned}
$$


When the initial conditions are $c_{a}(0)=1$ and $\dot{c}_{a}(0)=0$, then $c_{a}$ and $c_{b}$ can be shown to first order by direct integration of Equations 39 and 40,

$$
\begin{aligned}
& c_{a}(t)=1 \\
& c_{b}(t)=-\frac{i}{\hbar} \int_{0}^{t} H_{b a}^{\prime}\left(t^{\prime}\right) e^{i\left(E_{b}-E_{a}\right) t^{\prime} / \hbar} \mathrm{d} t^{\prime}
\end{aligned}
$$

\section{Fermi's Golden Rule}

Consider the system in the previous section introduced to a time harmonic perturbation of the form

$$
\hat{H}^{\prime}(\mathbf{r}, t)=H^{\prime}(\mathbf{r}) e^{-i \omega t}+H^{\prime \dagger}(\mathbf{r}) e^{i \omega t}
$$

where $H^{\prime \dagger}$ is the Hermitian conjugate of $H^{\prime}$ and $\omega$ is the angular frequency of the time harmonic perturbation. If the system begins in the intial state $a=i$, we can find the probability of a transition to state $b=f$ by solving, through direct integration, Equation 42:

$$
\begin{aligned}
c_{f} & =-\frac{i}{\hbar} \int_{0}^{t}\left(H_{f i}^{\prime} e^{-i \omega t}+H_{f i}^{\prime \dagger} e^{i \omega t}\right) e^{i\left(E_{f}-E_{i}\right) t^{\prime} / \hbar} \mathrm{d} t^{\prime} \\
& =\frac{-1}{\hbar}\left(H_{f i}^{\prime} \frac{e^{i\left(\omega_{f i}-\omega\right) t}-1}{\omega_{f i}-\omega}+H_{f i}^{\prime \prime} \frac{e^{i\left(\omega_{f i}+\omega\right) t}-1}{\omega_{f i}+\omega}\right)
\end{aligned}
$$

where $\omega_{f i}=\left(E_{f}-E_{i}\right) / \hbar$. Considering only drive frequencies close to the system's resonant frequency (i.e., $\omega \approx \omega_{f i}$ ), the probability of finding the state in $f$ after the perturbation is

$$
\left|c_{f}\right|^{2} \simeq \frac{4\left|H_{f i}^{\prime}\right|^{2}}{\hbar^{2}} \frac{\sin ^{2}\left[\left(\omega_{f i}-\omega\right) t / 2\right]}{\left(\omega_{f i}-\omega\right)^{2}}+\frac{4\left|H_{f i}^{\prime}\right|^{2}}{\hbar^{2}} \frac{\sin ^{2}\left[\left(\omega_{f i}+\omega\right) t / 2\right]}{\left(\omega_{f i}+\omega\right)^{2}}
$$

where the cross terms have been dropped because they are small compared to the above terms. Assuming that the measurement time is much greater than 
the perturbation time ( i.e., $\omega t \rightarrow \infty$ ), we can use the limit

$$
\lim _{t \rightarrow \infty} \frac{\sin ^{2}(x t / 2)}{x^{2}}=\frac{\pi t}{2} \delta(x)
$$

where $\delta(x)$ is the Dirac delta function, which simplifies Equation 45 to

$$
\left|c_{f}\right|^{2} \simeq \frac{2 \pi t}{\hbar^{2}}\left|H_{f i}^{\prime}\right|^{2} \delta\left(\omega_{f i}-\omega\right)+\frac{2 \pi t}{\hbar^{2}}\left|H_{f i}^{\prime}\right|^{2} \delta\left(\omega_{f i}+\omega\right)
$$

Using the property of the Dirac delta function, $\frac{1}{a} \delta(x)=\delta(a x)$, we can then calculate the transition rate from state $i$ to state $f$ as

$$
R_{i \rightarrow f}=\frac{\mathrm{d}}{\mathrm{d} t}\left|c_{f}\right|^{2}=\frac{2 \pi}{\hbar}\left|H_{f i}^{\prime}\right|^{2} \delta\left(E_{f}-E_{i}-\hbar \omega\right)+\frac{2 \pi}{\hbar}\left|H_{f i}^{\prime}\right|^{2} \delta\left(E_{f}-E_{i}+\hbar \omega\right)
$$

where the first term on the right corresponds to the absorption of light, because $E_{f}=E_{i}+\hbar \omega$, and the second term on the right corresponds to the emission of light, because $E_{f}=E_{i}-\hbar \omega$ (Figure 5).
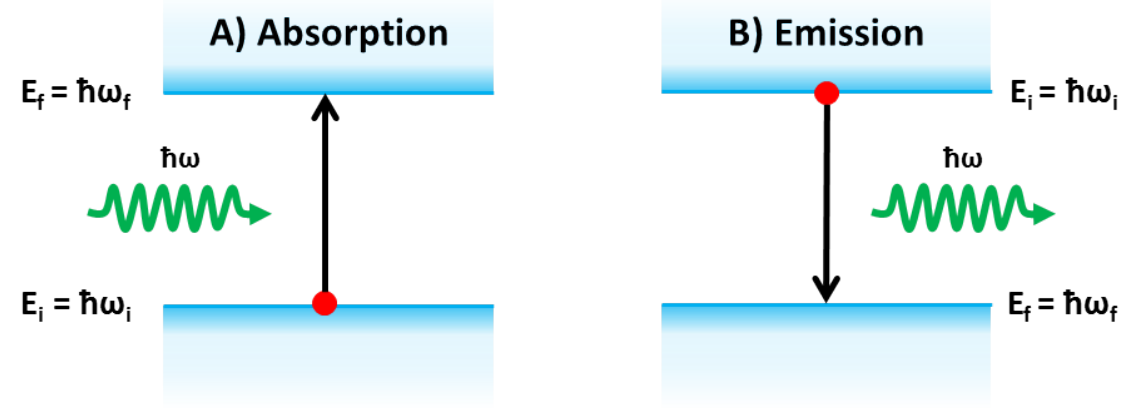

Figure 5: Diagram depicting $A$ ) the absorption and B) the emission of light in a two state system predicted by Fermi's golden rule (Equation 47).

\section{Absorption and Emission of Light}

The absorption and spontaneous emission of light in a semiconductor can be calculated using Fermi's golden rule (Equation 47) applied to interband transitions. The transition rate per unit volume $\left(\mathrm{s}^{-1} \mathrm{~cm}^{-3}\right)$ from an initial state, 
$a$, to an excited state, $b$, for the absorption of a photon is given using the first part of Equation 47,

$$
R_{a \rightarrow b}=\frac{2}{V} \frac{2 \pi}{\hbar}\left|H_{b a}^{\prime}\right|^{2} \delta\left(E_{b}-E_{a}-\hbar \omega\right) f_{a}\left(1-f_{b}\right)
$$

while the transition rate per unit volume for the emission of a photon is given using the second part of Equation 47,

$$
R_{b \rightarrow a}=\frac{2}{V} \frac{2 \pi}{\hbar}\left|H_{b a}^{\prime}\right|^{2} \delta\left(E_{a}-E_{b}+\hbar \omega\right) f_{b}\left(1-f_{a}\right)
$$

where the factor 2 takes into account spin degeneracy, $V$ is the volume, $f_{n}$ is the probability that state $n$ is occupied, and $\left(1-f_{n}\right)$ is the probability that state $n$ is unoccupied. It is assumed that the carriers follow Fermi-Dirac statistics, where

$$
f_{n}=\frac{1}{1+e^{\left(E_{n}-E_{F}\right) / k_{B} T}}
$$

with $E_{n}$ being the energy level of state $n$ and $E_{F}$ being the Fermi level of the device.

The net recombination rate is then the difference of Equations 48 and 49:

$$
\begin{aligned}
R & =R_{b \rightarrow a}-R_{a \rightarrow b} \\
& =\frac{2}{V} \frac{2 \pi}{\hbar}\left|H_{b a}^{\prime}\right|^{2} \delta\left(E_{b}-E_{a}-\hbar \omega\right)\left(f_{b}-f_{a}\right)
\end{aligned}
$$

where $\delta(-x)=\delta(x)$ is used.

The quantities $\left|H_{b a}^{\prime}\right|^{2}$ are found using the electron-photon interaction 
Hamiltionian in a periodic potential $U(\mathbf{r})$ :

$$
\begin{aligned}
\hat{H} & =\frac{1}{2 m}(\hat{\mathbf{p}}-q \mathbf{A})^{2}+U(\mathbf{r}) \\
& =\frac{p^{2}}{2 m}-\frac{q}{2 m}(\hat{\mathbf{p}} \cdot \mathbf{A}+\mathbf{A} \cdot \hat{\mathbf{p}})+\frac{q^{2} A^{2}}{2 m}+U(\mathbf{r}) \\
& \simeq H^{0}+H^{\prime}
\end{aligned}
$$

where $q$ is the charge of the electron; $\mathbf{A}$ is the magnetic vector potential; $H^{0}=p^{2} / 2 m+U(\mathbf{r})$ is the unperturbed Hamiltonian; and

$$
H^{\prime}=-\frac{q}{2 m} \mathbf{A} \cdot \hat{\mathbf{p}}
$$

is the perturbed Hamiltonian where the Coulomb gauge, $\nabla \cdot \mathbf{A}=0$, is used to remove the first cross term in Equation 53, and the approximation $|q \mathbf{A}| \ll|\hat{\mathbf{p}}|$ is used to remove the quadratic term. The use of optical wavelengths $(\sim 500 \mathrm{~nm})$ in an LED QW ( $\sim 3 \mathrm{~nm})$ also justifies the approximation that $\mathbf{A}(\mathbf{r})=\mathbf{A}_{0} e^{i \mathbf{k}_{\gamma} \cdot \mathbf{r}} \simeq \mathbf{A}$, where $\mathbf{A}$ is a constant vector orthogonal to the direction of travel and $\mathbf{k}_{\gamma}$ is the photon wave vector.

To find $H_{b a}^{\prime}$ in an LED QW, we employ Bloch wavefunctions (Equation 30) of the form

$$
\psi_{a}(\mathbf{r})=u_{v}(\mathbf{r}) \frac{e^{i \mathbf{k}_{t} \cdot \mathbf{r}}}{\sqrt{A}} p_{j}(z)
$$

for a hole in the valence band with an envelope wavefunction $p_{j}(z)$, and of the form

$$
\psi_{b}(\mathbf{r})=u_{c}(\mathbf{r}) \frac{e^{i \mathbf{k}_{t}^{\prime} \cdot \mathbf{r}}}{\sqrt{A}} n_{i}(z)
$$

for an electron in the conduction band with an envelope wavefunction $n_{i}(z)$, where $\mathrm{k}_{t}$ and $\mathrm{k}_{t}^{\prime}$ are the wave vectors in the transverse $\mathrm{xy}$-direction for the hole and electron, respectively, and the non-boldface $A$ is the area in the transverse 
direction. Combining Equation 54 with Equations 55 and 56 gives

$$
\begin{aligned}
H_{b a}^{\prime} & =\int \psi_{b}^{*} H^{\prime} \psi_{a} \mathrm{~d} V \\
& =-\frac{q \mathbf{A}}{2 m} \cdot \int u_{c}^{*} n_{i}^{*}\left[\frac{\hbar}{i} \nabla u_{v} p_{j}+\hbar \mathbf{k}_{t} u_{v} p_{j}+\frac{\hbar}{i} u_{v} \frac{\partial}{\partial z} p_{j} \hat{\mathbf{z}}\right] e^{i\left(\mathbf{k}_{t}-\mathbf{k}_{t}^{\prime}\right) \cdot \mathbf{r}} \frac{\mathrm{d} V}{A} \\
& \simeq-\frac{q \mathbf{A}}{2 m} \cdot \int_{\Omega} u_{c}^{*}\left(\frac{\hbar}{i} \nabla\right) u_{v} \mathrm{~d} \Omega \int_{A} e^{i\left(\mathbf{k}_{t}-\mathbf{k}_{t}^{\prime}\right) \cdot \mathbf{r}} \frac{\mathrm{d} A}{A} \int_{z} n_{i}^{*} p_{j} \mathrm{~d} z \\
& =-\frac{q}{2 m} \mathbf{A} \cdot \mathbf{p}_{c v} \delta_{\mathbf{k}_{t} \mathbf{k}_{t}^{\prime}}\left\langle n_{i} \mid p_{j}\right\rangle
\end{aligned}
$$

where the boldface $\mathbf{p}_{c v}$ denotes the momentum matrix elements coupling the conduction and valence band bulk wavefunctions, $\Omega$ is the volume of a unit cell, and Equation 57 uses the approximation that $n_{i}, p_{j}$, and $e^{i \mathbf{k}_{t} \cdot \mathbf{r}}$ are constant over a unit cell. The term $\left\langle n_{i} \mid p_{j}\right\rangle$ is known as the overlap integral for the electron and hole wavefunctions in states $i$ and $j$, respectively. From Equation 58 we can calculate the net recombination rate when light is present within an LED QW: 22

$$
R=\frac{\pi q^{2}}{2 m^{2} \hbar} \frac{2}{V} \sum_{i, j}\left|\left\langle n_{i} \mid p_{j}\right\rangle\right|^{2} \sum_{\mathbf{k}_{t}}\left|\mathbf{A} \cdot \mathbf{p}_{c v}\right|^{2} \delta\left(E_{b}-E_{a}-\hbar \omega\right)\left(f_{b}-f_{a}\right)
$$

where

$$
\begin{aligned}
& E_{a}=E_{h j}-\frac{\hbar^{2} k_{t}^{2}}{2 m_{h}^{*}} \\
& E_{b}=E_{g}+E_{e i}+\frac{\hbar^{2} k_{t}^{2}}{2 m_{e}^{*}}
\end{aligned}
$$

are the total energies of states $a$ and $b$ as functions of the hole energy $E_{h j}$ and the electron energy $E_{e i}$, where the $i$ and $j$ subscripts refer to the energies of the allowed states in the quantum well. 


\subsubsection{Semi-Classical Drift-Diffusion Model}

For an electron (n-type) carrier density, $n$, with current density, $\mathbf{J}_{n}$, and a hole ( $\mathrm{p}$-type) carrier density, $p$, with current density, $\mathbf{J}_{p}$, the carrier transport equations are

$$
\begin{aligned}
& \mathbf{J}_{n}=q \mu_{n} n \mathbf{E}+q D_{n} \nabla n \\
& \mathbf{J}_{p}=q \mu_{p} p \mathbf{E}-q D_{p} \nabla p
\end{aligned}
$$

where $\mu_{n}, \mu_{p}$ are the respective carrier mobilities and $D_{n}, D_{p}$ are the respective carrier diffusion coefficients. The total current density $\mathbf{J}=\mathbf{J}_{n}+\mathbf{J}_{p}$ must then follow the carrier continuity equation for semiconductors (refer to Equation 5):

$$
\nabla \cdot\left(\mathbf{J}_{p}+\mathbf{J}_{n}\right)+q \frac{\partial}{\partial t}(p-n)=0
$$

Since the electrons and holes are independent of each other, this equation can be separated into two differential equations separated by a constant:

$$
\begin{aligned}
& \nabla \cdot \mathbf{J}_{n}-q \frac{\partial n}{\partial t}=+q R \\
& \nabla \cdot \mathbf{J}_{p}+q \frac{\partial p}{\partial t}=-q R
\end{aligned}
$$

where $R$ is the net recombination rate of non-equilibrium carriers such that

$$
R=R_{n}-G_{n}
$$

for electrons and

$$
R=R_{p}-G_{p}
$$


for holes with $R_{n}, R_{p}$ being the recombination rates and $G_{n}, G_{p}$ being the generation rates for their respective $n$-type or $p$-type non-equilibrium carriers.

At equilibrium the carrier concentrations are constant such that $\dot{n}=\dot{p}=0$. If the net recombination rate across an active region of width $w$ is constant, then

$$
\begin{aligned}
& J_{n}=q w R=q w\left(R_{n}-G_{n}\right) \\
& J_{p}=-q w R=-q w\left(R_{p}-G_{p}\right)
\end{aligned}
$$

A phenomenological approach, along with the quantum mechanical approach (section 1.4.2), is used in SiLENSe ${ }^{24}$ to model electron and hole net recombination rates and to develop the non-equilibrium current generated within the LED device's active region.

\section{Interband Radiative Recombination}

Band to band optical transitions occur through two different processes: electron-hole pair generation and electron-hole pair recombination (Figure 6).

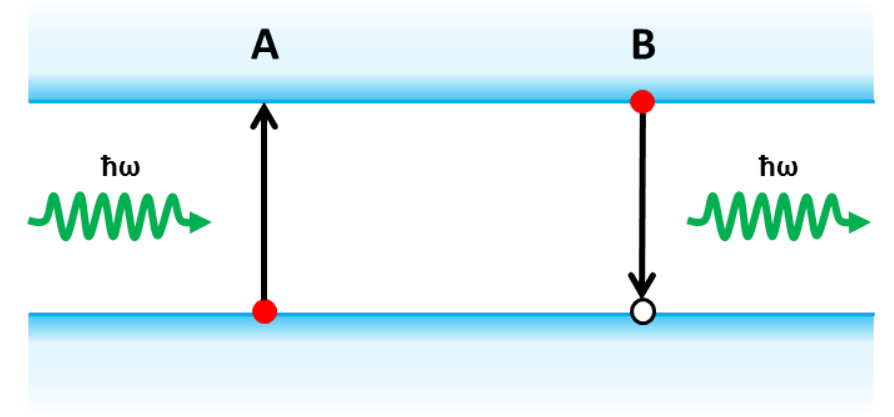

Figure 6: Depiction of the interband radiative transition with $(A)$ the generation of an electron-hole pair and (B) the recombination of an electron-hole pair. 
This process can be described by one chemical rate equation:

$$
\mathrm{n}+\mathrm{p} \underset{e}{\stackrel{c}{\rightleftharpoons}} \gamma
$$

where $\gamma$ signifies a photon created, $c$ is the capture rate, and $e$ is the emission rate of the electron-hole pair. This gives the generation and recombination rates

$$
R_{n}=R_{p}=c n p G_{n}=G_{p}=e
$$

where it is assumed that each photon absorbed creates an electron-hole pair. The net recombination rate will be zero at equilibrium, giving

$$
\begin{aligned}
R & =c n_{0} p_{0}-e=0 \\
\Rightarrow e & =c n_{0} p_{0}
\end{aligned}
$$

The net recombination rate is then 22,24

$$
\begin{aligned}
R & =c\left(n p-n_{0} p_{0}\right) \\
& =c n p\left(1-e^{-\left(F_{n}-F_{p}\right) / k_{B} T}\right)
\end{aligned}
$$

where the Boltzmann distribution is assumed for the carriers:

$$
\begin{aligned}
& n=N_{n} \exp \left[-\frac{E_{c}-F_{n}}{k_{B} T}\right] \\
& p=N_{p} \exp \left[-\frac{F_{p}-E_{v}}{k_{B} T}\right]
\end{aligned}
$$

where $N_{n}, N_{p}$ are the effective doping concentrations and $F_{n}, F_{p}$ are the quasi Fermi levels for the electrons and holes, respectively, and are equal to the Fermi level at equilibrium: $F_{n}=F_{p}=E_{F}$ (at equilibrium). Note that the band to band radiative transition rate is proportional to the square of the carrier 
concentration.

\section{Shockley-Read-Hall Recombination}

Shockley-Read-Hall $(\mathrm{SRH})$ recombination involves the interaction of charge carriers with trap states in the semiconductor. This involves four different processes: electron capture, electron emission, hole capture, and hole emission (Figure 7).

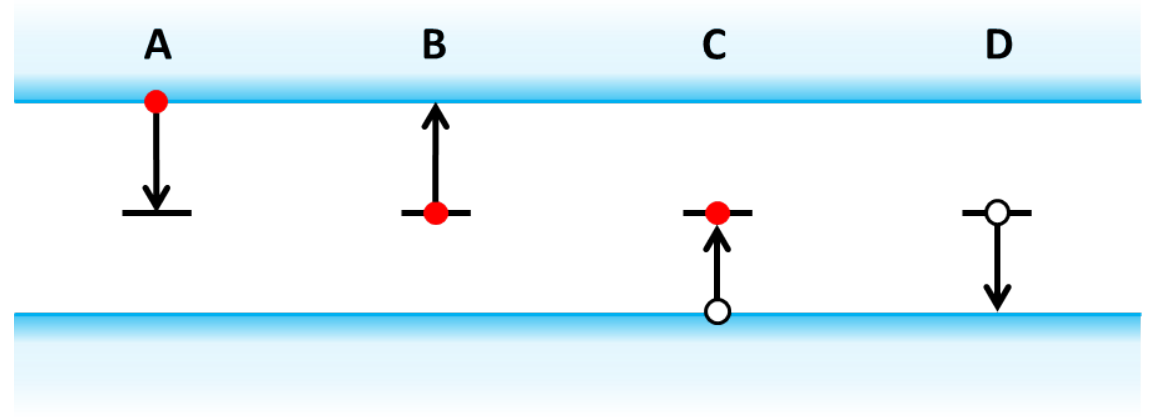

Figure 7: Depiction of $\mathrm{SRH}$ non-radiative transitions with $(A)$ electron capture, (B) electron emission, (C) hole capture, and (D) hole emission.

These four processes are written as two equilibrium rate equations for electron processes and hole processes:

$$
\begin{aligned}
& \mathrm{n}+\mathrm{t}_{\circ} \underset{e_{n}}{\stackrel{c_{n}}{\rightleftharpoons}} \mathrm{t}_{\bullet} \\
& \mathrm{p}+\mathrm{t}_{\bullet} \underset{e_{p}}{\stackrel{c_{p}}{\rightleftharpoons}} \mathrm{t}_{\circ}
\end{aligned}
$$

where $t_{\bullet}$ is the concentration of filled traps and $t_{\circ}$ is the concentration of open traps. This gives the generation and recombination rates

$$
\begin{array}{ll}
R_{n}=c_{n} n N_{t}\left(1-f_{t}\right) & G_{n}=e_{n} N_{t} f_{t} \\
R_{p}=c_{p} p N_{t} f_{t} & G_{p}=e_{p} N_{t}\left(1-f_{t}\right)
\end{array}
$$

where $N_{t}=t_{\bullet}+t_{\circ}$ is the total concentration of traps, $f_{t}$ is the proportion of 
occupied traps, and $\left(1-f_{t}\right)$ is the proportion of unoccupied traps. Setting the net recombination rate to zero at equilibrium gives

$$
\begin{aligned}
& e_{n}=c_{n}\left(n_{0} \frac{1-f_{t_{0}}}{f_{t_{0}}}\right)=c_{n} n_{1} \\
& e_{p}=c_{p}\left(p_{0} \frac{f_{t_{0}}}{1-f_{t_{0}}}\right)=c_{p} p_{1}
\end{aligned}
$$

where $n_{1}$ and $p_{1}$ have been introduced for convenience. At equilibrium, $R_{n}-G_{n}=R_{p}-G_{p}$, giving

$$
f_{t}=\frac{c_{n} n+c_{p} p_{1}}{c_{n}\left(n+n_{1}\right)+c_{p}\left(p+p_{1}\right)}
$$

Plugging Equation 83 back into the net recombination rate gives 22,24

$$
\begin{aligned}
R_{n}-G_{n}=R_{p}-G_{p} & =N_{t}\left[\frac{c_{n} c_{p} n p-e_{n} e_{p}}{c_{n}\left(n+n_{1}\right)+c_{p}\left(p+p_{1}\right)}\right] \\
& =\left[\frac{n+n_{1}}{N_{t} c_{p}}+\frac{p+p_{1}}{N_{t} c_{n}}\right]^{-1} n p\left(1-e^{-\left(F_{n}-F_{p}\right) / k_{B} T}\right)
\end{aligned}
$$

where $n_{1} p_{1}=n_{0} p_{0}$ is used along with Equations 75 and 76 . Note that the $\mathrm{SRH}$ recombination rate (Equation 84 ) is proportional to the carrier concentration.

\section{Auger Recombination}

Auger electronic transitions can either be a band to trap state process or a band to band process. The band to trap state transitions involve eight different processes similar to the four SRH processes, except that for each SRH process a secondary electron or hole is involved (Figure 8). 

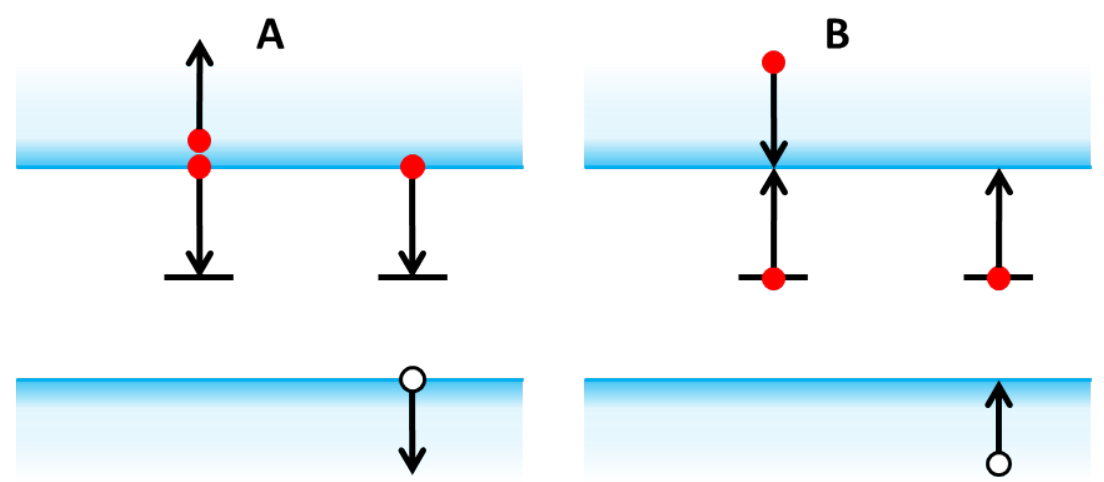

C

D
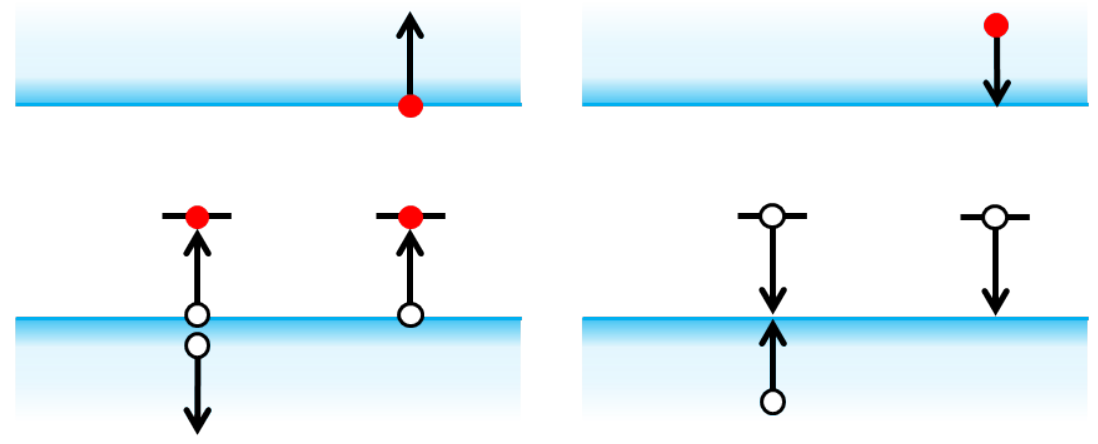

Figure 8: Depiction of Auger non-radiative trap state transitions with (A) electron capture with electron excitation (left) and hole excitation (right), (B) electron emission with excited electron (left) and excited hole (right), (C) hole capture with hole excitation (left) and electron excitation (right), and (D) hole emission with excited hole (left) and excited electron (right).

These processes have four different chemical rate equations:

$$
\begin{aligned}
& 2 \mathrm{n}+\mathrm{t}_{\circ} \underset{e_{n}^{n}}{\stackrel{c_{n}^{n}}{\leftrightarrows}} \mathrm{n}+\mathrm{t} . \\
& \mathrm{n}+\mathrm{p}+\mathrm{t}_{\circ} \underset{e_{n}^{p}}{\stackrel{c_{n}^{p}}{p}} \mathrm{p}+\mathrm{t} .
\end{aligned}
$$

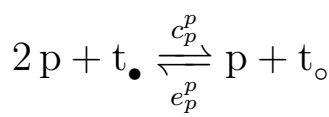

$$
\begin{aligned}
& \mathrm{n}+\mathrm{p}+\mathrm{t} \cdot \underset{e_{p}^{n}}{\stackrel{c_{p}^{n}}{p}} \mathrm{n}+\mathrm{t} 。
\end{aligned}
$$


which produce the recombination and generation rates:

$$
\begin{aligned}
& R_{n}=\left(c_{n}^{n} n+c_{n}^{p} p\right) n N_{t}\left(1-f_{t}\right)=c_{n} n N_{t}\left(1-f_{t}\right) \\
& G_{n}=\left(e_{n}^{n} n+e_{n}^{p} p\right) N_{t} f_{t}=e_{n} N_{t} f_{t} \\
& R_{p}=\left(c_{p}^{n} n+c_{p}^{p} p\right) p N_{t} f_{t}=c_{p} p N_{t} f_{t} \\
& G_{p}=\left(e_{p}^{n} n+e_{p}^{p} p\right) N_{t}\left(1-f_{t}\right)=e_{p} N_{t}\left(1-f_{t}\right)
\end{aligned}
$$

where $c_{n}, e_{n}, c_{p}$, and $e_{p}$ were introduced to show the similarity with the $\mathrm{SRH}$ processes (Equations 79 and 80). Therefore, the net recombination rate for Auger trap state transitions is given by the $\mathrm{SRH}$ net recombination rate (Equation 84) when the appropriate rate constants are substituted.

The more commonly discussed Auger transitions are those between the conduction and valence bands. There are four processes involved for band to band Auger non-radiative recombination that are similar to those of band to band radiative transitions, except that an electron or hole is excited instead of a photon (Figure 9).

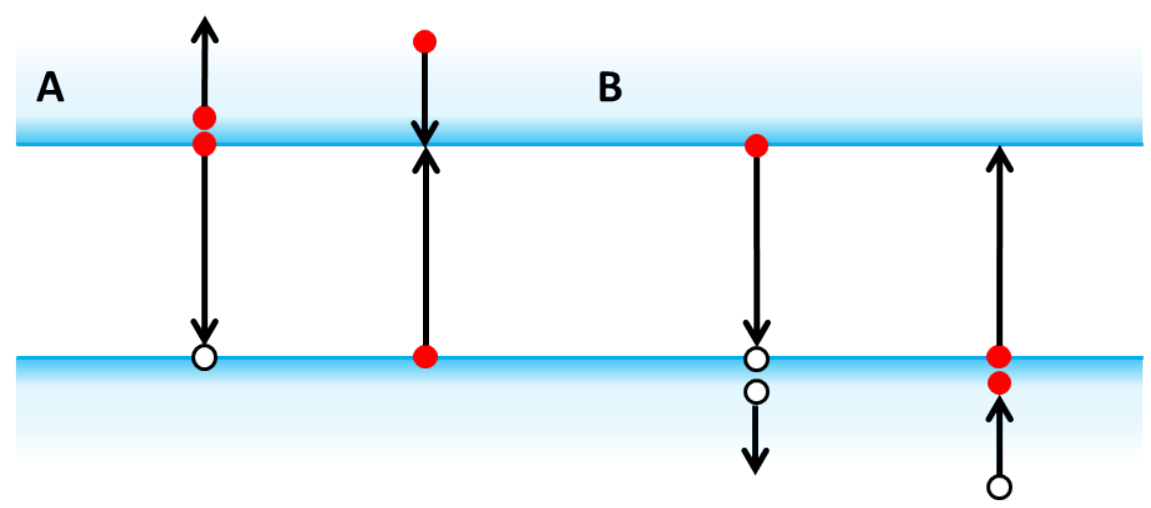

Figure 9: Depiction of Auger non-radiative band to band state transitions showing (A) electron capture (left) and emission (right) with an excited electron, and (B) electron capture (left) and emission (right) with an excited hole. 
The chemical rate equations for these transitions are

$$
\begin{aligned}
& 2 \mathrm{n}+\mathrm{p} \underset{e_{n}^{A U}}{\stackrel{c_{n}^{A U}}{\rightleftharpoons}} \mathrm{n} \\
& \mathrm{n}+2 \mathrm{p} \underset{e_{p}^{A U}}{\stackrel{c_{p}^{A U}}{\rightleftharpoons}} \mathrm{p}
\end{aligned}
$$

which give the following recombination and generation rates:

$$
\begin{array}{ll}
R_{n}=c_{n}^{A U} n^{2} p & G_{n}=e_{n}^{A U} n \\
R_{p}=c_{p}^{A U} n p^{2} & G_{p}=e_{p}^{A U} p
\end{array}
$$

The net recombination rates, $R_{n}-G_{n}=c_{n}^{A U} n\left(n p-n_{0} p_{0}\right)$ and $R_{p}-G_{p}=c_{p}^{A U} p\left(n p-n_{0} p_{0}\right)$, are obtained from using $R=0$ at equilibrium. The total band to band Auger recombination rate is then the total of the two net recombination rates because they each involve the creation and annihilation of electron-hole pairs: ${ }^{22,24}$

$$
\begin{aligned}
R_{\text {total }}^{A U} & =R_{n}-G_{n}+R_{p}-G_{p} \\
& =\left(c_{n}^{A U} n+c_{p}^{A U} p\right)\left(n p-n_{0} p_{0}\right) \\
& =\left(c_{n}^{A U} n+c_{p}^{A U} p\right) n p\left(1-e^{-\left(F_{n}-F_{p}\right) / k_{B} T}\right)
\end{aligned}
$$

Note that the Auger recombination rate $R^{A U}$ is proportional to the cube of the carrier concentration.

\section{The ABC Model}

At equilibrium the total number of carriers in a LED QW does not change, and any current injected into the active region must follow Equations 69 and 70. When considering the carrier dependence of Equations 74, 84, and 97, the net recombination rate inside an LED QW is often written in the more simplistic 
form:

$$
R=A n+B n^{2}+C n^{3}
$$

where $n$ is the carrier concentration, $A$ is the $\mathrm{SRH}$ recombination coefficient, $B$ is the radiative recombination coefficient, and $C$ is the Auger recombination coefficient. Equation 98 is called the "ABC model" of carrier recombination.

The current inside an LED QW using Equations 69, 70, and 98 then becomes

$$
J=q w\left(A n+B n^{2}+C n^{3}\right)
$$

The internal quantum efficiency (IQE), $\eta_{I Q E}$, of a semiconductor LED device is then defined by

$$
\eta_{I Q E} \equiv \frac{J^{R A D}}{J}=\frac{B n^{2}}{A n+B n^{2}+C n^{3}}
$$

Graphing $\eta_{I Q E}$ against current density therefore leads to the droop problem commonly seen in group-III nitride devices due to the $n^{3}$ dependence of Auger recombination.

\section{Methods and Materials}

\subsection{Device Design}

An $\ln _{0.2} \mathrm{Ga}_{0.8} \mathrm{~N} / \mathrm{GaN}$ MQW device with a $470 \mathrm{~nm}$ peak wavelength was simulated with SiLENSe. The structure was grown on a $0.3 \mu \mathrm{m}$ n-type GaN substrate $\left(\mathrm{n}\right.$-doping $\left.=7 \times 10^{18} \mathrm{~cm}^{-3}\right)$. The original device's active region 
consisted of three $3 \mathrm{~nm} \operatorname{In}_{0.2} \mathrm{Ga}_{0.8} \mathrm{~N}$ QWs separated by two $10 \mathrm{~nm}$ GaN barrier layers, which all had an intrinsic n-doping concentration of $2 \times 10^{17}$ due to hydrogen impurities. Multiple devices were developed with varying $\mathrm{In}_{0.2} \mathrm{Ga}_{0.8} \mathrm{NQW}$ composition profiles (discussed below). This was followed by a $10 \mathrm{~nm}$ p-type $\mathrm{GaN}$ barrier layer ( $\mathrm{p}$-doping $=6 \times 10^{18}$ ), a $10 \mathrm{~nm}$ p-type $\mathrm{Al}_{0.2} \mathrm{Ga}_{0.8} \mathrm{~N}$ EBL ( $\mathrm{p}$-doping $=3 \times 10^{19}$ ), and finally a $0.2 \mu \mathrm{m}$ p-type $\mathrm{GaN}$ capping layer $\left(p\right.$-doping $\left.=2 \times 10^{19}\right)$. This structure can be seen in Figure 10 .

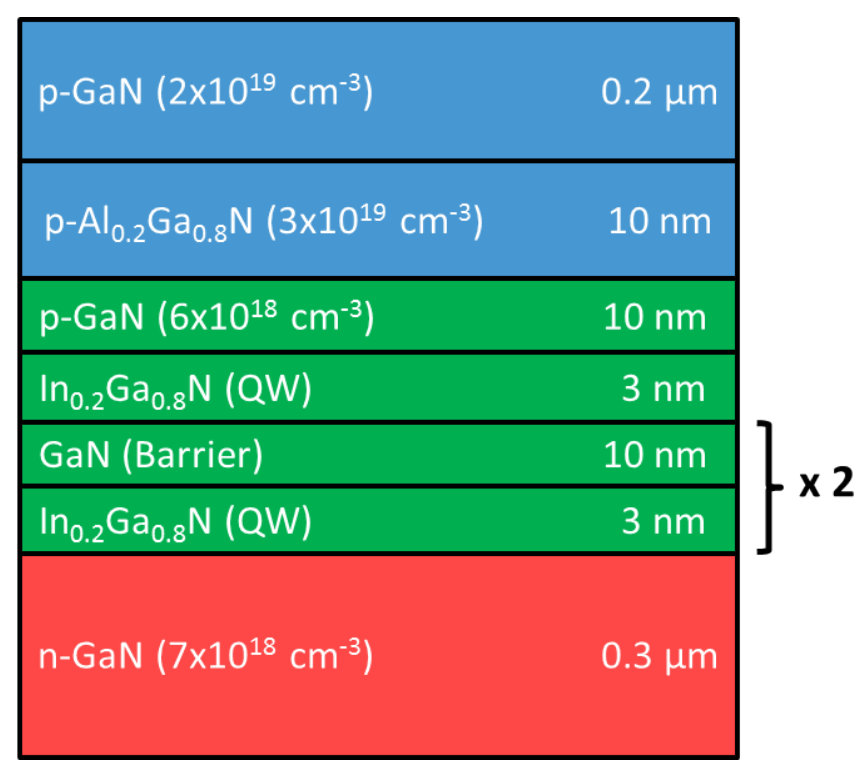

Figure 10: Picture of the layer structure for the InGaN/GaN MQW heterostructure employed in SiLENSe simulations.

\subsubsection{Internal Structure}

In order to simulate a more realistic InGaN QW composition profile, ${ }^{4,25}$ a series of trapezoidal and triangular wells was used (Figure 11). Although a Gaussian or skewed Gaussian indium distribution would preferably be employed, ${ }^{4,25}$

SiLENSe is unable to simulate such a model. Therefore, the trapezoidal and triangular well model described herein is to be used as more of a qualitative rather than a quantitative model. In order to compare the effect of well shape to changes in polarization, another set of InGaN/GaN square MQW heterostructure devices was developed with the polarization constants in 
Table I reduced to $70 \%$ and $40 \%$ of their original values.

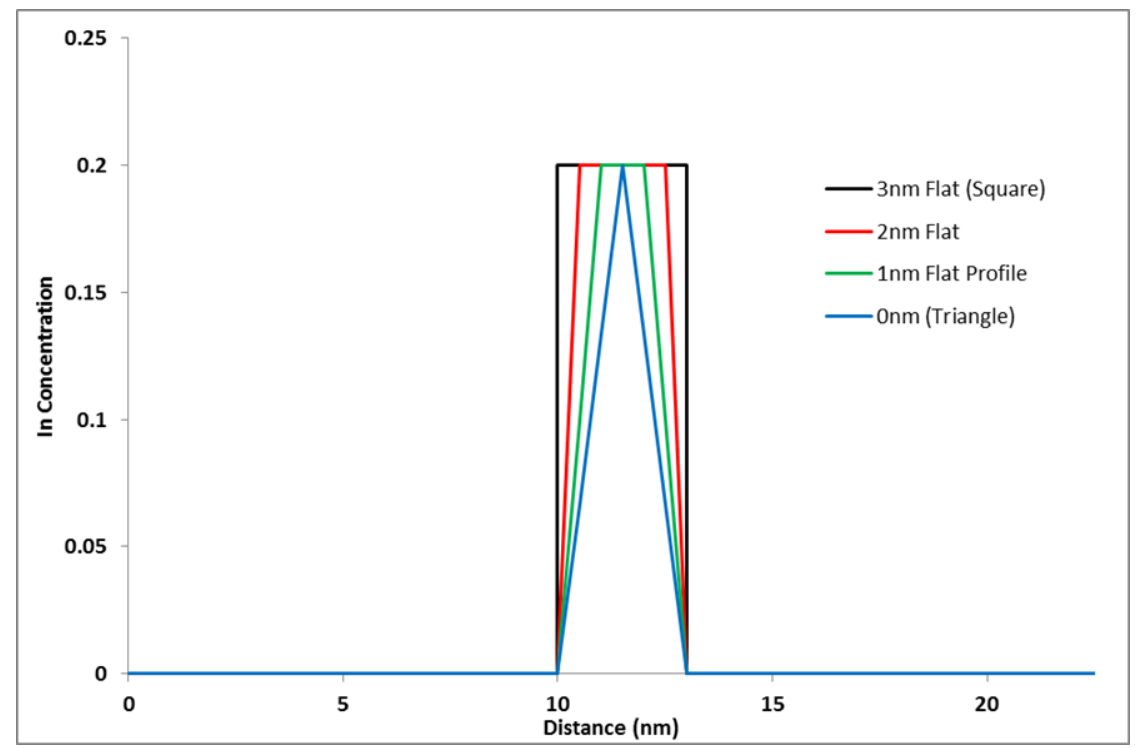

Figure 11: Graphical representation of indium concentration across one QW for the $3 \mathrm{~nm}$ (flat), $2 \mathrm{~nm}, 1 \mathrm{~nm}$, and $0 \mathrm{~nm}$ (triangular) devices. These are to be used as simple and qualitative representations of a non square QW as shown in Kisielowski et al. ${ }^{4}$

\subsection{Measurement Parameters}

The different InGaN/GaN MQW heterostructure devices were simulated under a varying bias voltage ranging from $0-4.5 \mathrm{~V}$. The current density, J, was recorded across the entire device, and a J-V curve was tabulated for each of the $\mathrm{In}_{0.2} \mathrm{Ga}_{0.8} \mathrm{NQW}$ composition profiles as well as polarization constant values. The current density of the devices was used because it directly indicates a simulation's ability to provide accurate experimental results. Furthermore, previous studies stated that reduced polarization constants were required to obtain experimental currents. The shapes of the J-V curves for all the simulated devices were compared in order to determine similarities between reduced polarization and QW shape. These similarities in $\mathrm{J}-\mathrm{V}$ characteristics were then compared to the band structure and electron-hole behavior of each device in order to determine any internal trends. 


\section{Results}

\subsection{Effect of Polarization Reduction}

The J-V characteristic of the original (100\% polarization) $\ln _{0.2} \mathrm{Ga}_{0.8} \mathrm{~N} / \mathrm{GaN} 3 \mathrm{QW}$ heterostructure shows a high onset voltage, requiring $4.0 \mathrm{~V}$ to achieve a current density of $10 \mathrm{~A} / \mathrm{cm}^{2}$ (Figure 12). This result does not match empirical evidence since experimental $3 Q W$ InGaN/GaN devices have onset voltages closer to $3.0 \mathrm{~V} .{ }^{26,27}$ Reducing the $\ln _{0.2} \mathrm{Ga}_{0.8} \mathrm{~N}$ polarization coefficients to $70 \%$ and $40 \%$ significantly improves the onset voltage lowering it to $3.4 \mathrm{~V}$ and $3.0 \mathrm{~V}$, respectively. This shows that reduced polarization coefficients lead to dramatically improved theoretical results.

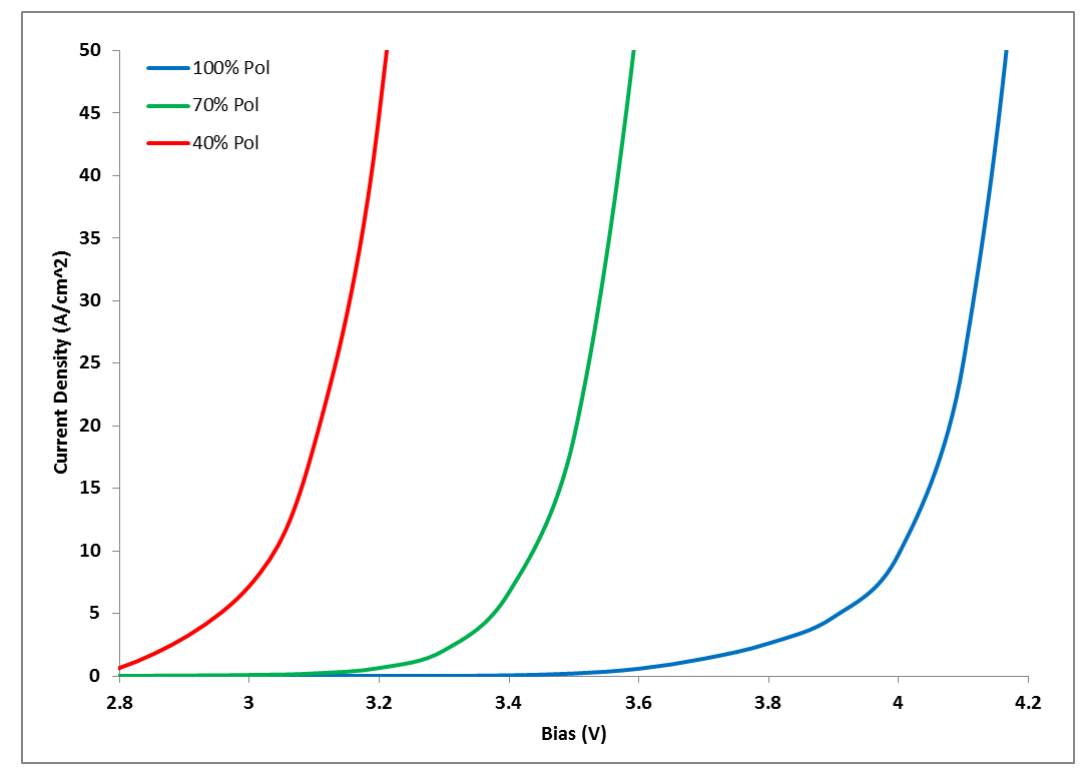

Figure 12: The current density vs. bias voltage of the $\ln _{0.2} \mathrm{Ga}_{0.8} \mathrm{~N} 3 \mathrm{QW}$ heterostructure for polarization coefficients at $100 \%$ (blue), $70 \%$ (green), and $40 \%$ (red) of their empirical values.

\subsubsection{Band Diagram with Reduced Polarization}

The band diagram of the $\ln _{0.2} \mathrm{Ga}_{0.8} \mathrm{~N} / \mathrm{GaN} 3 \mathrm{QW}$ heterostructure was analysed at a bias of $3.5 \mathrm{~V}$ for each of the reduced polarization constructs. The $100 \%$ polarization case shows significant linear band bending in both the $\ln _{0.2} \mathrm{Ga}_{0.8} \mathrm{~N}$ 
QW regions as well as in the GaN barrier regions (Figure 13). Nonlinear deviations are also seen in the $\mathrm{n}$ - and $\mathrm{p}$-type regions of the device due to the free charge carriers introduced by doping (Equation 14). The n-type GaN bends upwards while the p-type GaN bends downwards due to the space charge formed by the depletion region when the device was created. The p-type $\mathrm{Al}_{0.2} \mathrm{Ga}_{0.8} \mathrm{~N}$ and $\mathrm{GaN}$ layers show significant nonlinear band bending due to both polarization and free charge carrier effects. Slight nonlinear behavior is also seen in the $\mathrm{QW}$ and barrier regions due to the hydrogen impurity doping.

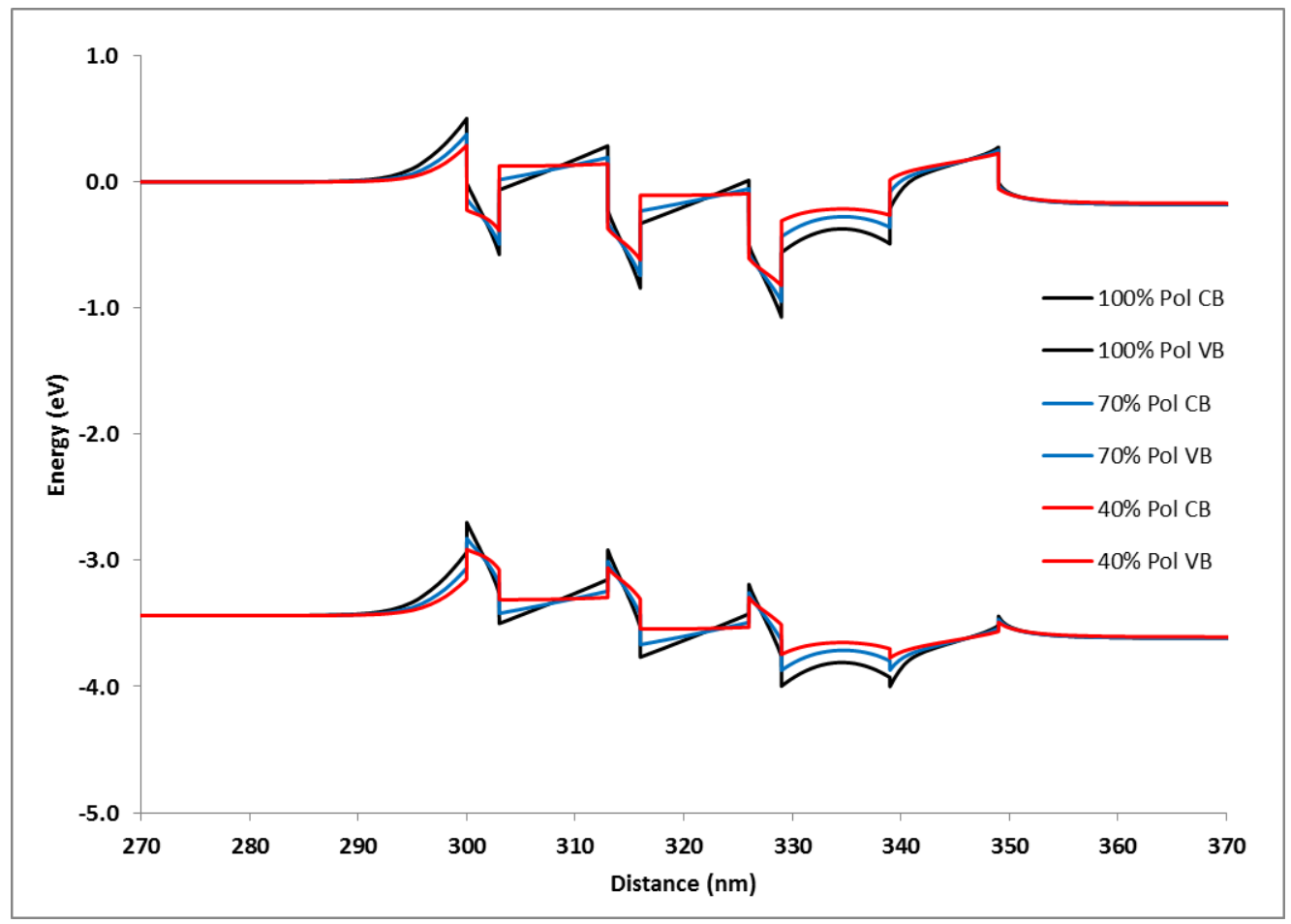

Figure 13: The band diagrams of the $\ln _{0.2} \mathrm{Ga}_{0.8} \mathrm{~N} 3 \mathrm{QW}$ heterostructure at a 3.5 $\checkmark$ bias for polarization coefficients at $100 \%, 70 \%$, and $40 \%$ of their empirical values. Reducing polarization causes band flattening in the $\mathrm{QW}$ and barrier regions. This increases the electron-hole overlap and subsequently increases current density across the device.

Band flattening is seen as the InGaN polarization coefficients are reduced. As the band structure in Figure 13 shifts to $70 \%$ and $40 \%$ polarization, both the $\mathrm{CB}$ and $\mathrm{VB}$ in the $\mathrm{QW}$ and barrier regions begin to flatten out. This flattening reduces the polarization induced electric field in the $\mathrm{QW}$ and barrier regions, 
which thereby decreases reverse current flow. Band flattening in the QW regions also raises the band energies on the $p$-type side, which inhibits electron flow while increasing hole flow through the device. This increases the QW hole concentration across the device, which leads to a higher electron-hole overlap

\subsubsection{Wavefunctions with Reduced Polarization}

A closer look at each $\ln _{0.2} \mathrm{Ga}_{0.8} \mathrm{~N}$ QW shows how polarization decreases the electron-hole overlap. Figure 14 graphs the first CB electron energy wavefunction (EE1) and the first VB heavy hole energy wavefunction ( $\mathrm{HH} 1)$ for the middle $\operatorname{In}_{0.2} \mathrm{Ga}_{0.8} \mathrm{~N}$ QW of each device. The $100 \%$ polarization case (Figure 14-A) shows how the polarization induced electric field forces the electron and hole wavefunctions to opposite sides of the well. As the polarization is reduced (Figures 14-B and 14-C), the band energy in the QW flattens out and allows the electron and hole wavefunctions to distribute more evenly across the well. Figure 14-D shows the overall effect as polarization is reduced, which reveals how the electron and hole wavefunctions shift closer to each other and how their spread increases as polarization is reduced. 


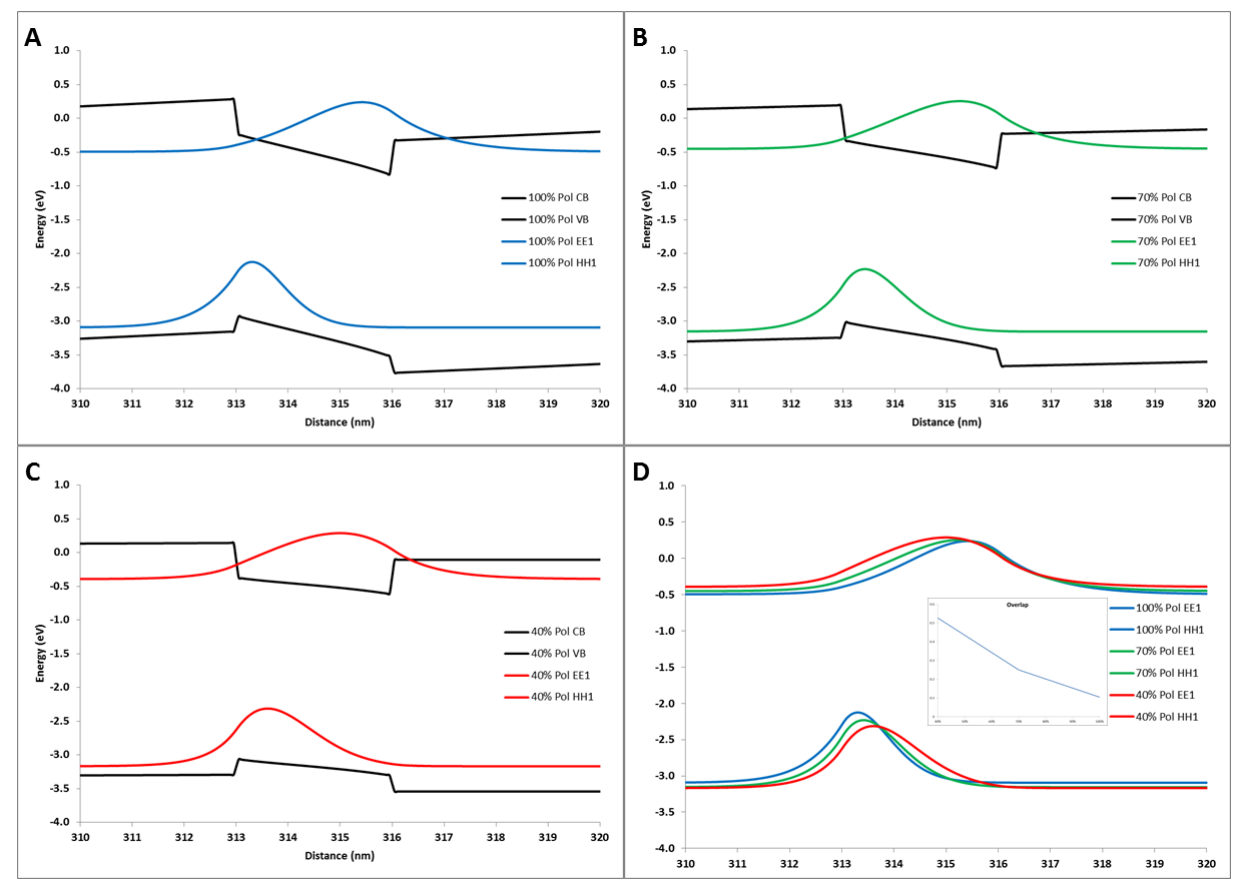

Figure 14: The electron-hole wavefunctions for the middle $Q W$ in the $\ln _{0.2} \mathrm{Ga}_{0.8} \mathrm{~N}$ $3 Q W$ heterostructure at a $3.5 \mathrm{~V}$ bias for polarization coefficients at (A) $100 \%$ (overlap $=10.4 \%$ ), (B) $70 \%$ (overlap $=25.0 \%$ ), and $(\mathrm{C}) 40 \%$ (overlap $=$ $52.7 \%$ ) of their empirical values. (D) This figure shows how the electronhole wavefunctions tend toward each other as polarization is reduced (inset graphically shows the trend with overlap). This increases the electron-hole overlap, which is in accordance with the increased current density calculated.

It is also important to note the slight increase in energy separation of the electron and hole wavefunctions (Figure 14-D) due to the quantum confined Stark effect. This effect is due to the change in the energy gap as polarization decreases, which can be seen in Figure 13. Therefore, reduction in polarization leads to a blue shift in the emission spectrum of the LED.

\subsection{Effect of Quantum Well Shape}

The original device with $3 \mathrm{~nm}$ square $\ln _{0.2} \mathrm{Ga}_{0.8} \mathrm{~N}$ QWs does not show behavior corresponding with experiment as discussed in the previous section. Alteration of the $3 \mathrm{~nm} \ln _{0.2} \mathrm{Ga}_{0.8} \mathrm{~N}$ QW shape to a trapezoid with base length of $1 \mathrm{~nm}$ or 0 $\mathrm{nm}$ (triangular) decreases the onset voltage from $4.0 \mathrm{~V}$ to $3.8 \mathrm{~V}$ or $3.5 \mathrm{~V}$, respectively. 


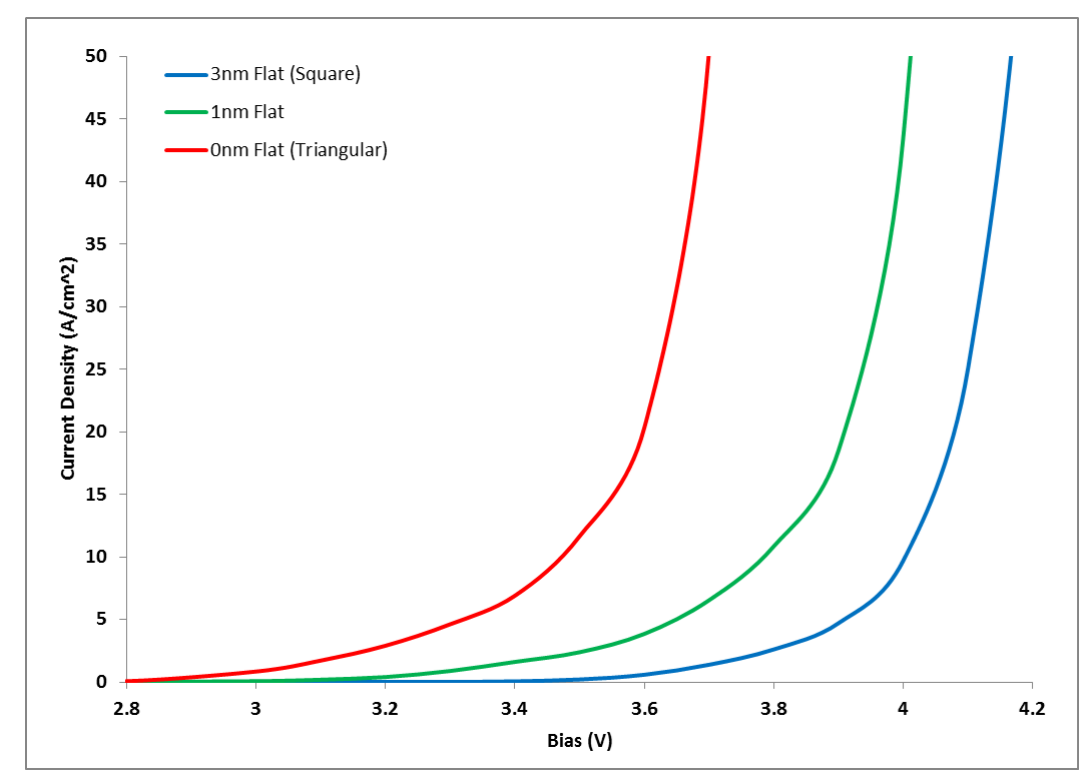

Figure 15: The current density vs. bias voltage of the $\ln _{0.2} \mathrm{Ga}_{0.8} \mathrm{~N} 3 \mathrm{QW}$ heterostructure for trapezoidal indium profiles with base lengths of $3 \mathrm{~nm}$ (blue), $1 \mathrm{~nm}$ (green), and $0 \mathrm{~nm}$ (red).

The shift of the $\mathrm{J}-\mathrm{V}$ curve to the left in Figure 15 is similar to the shift seen in Figure 12 because the mechanism for current stimulation in both cases involves an increase in electron-hole overlap. This is a direct result of changes in the overall band structure of the device.

\subsubsection{Band Diagram with Altered Quantum Well Shape}

Altering the indium composition profile has both a direct and secondary effect on the LED device band structure. The direct effect is seen in the shape of the InGaN QWs in Figure 16. As the flat $\ln _{0.2} \mathrm{Ga}_{0.8} \mathrm{~N}$ portion of the well narrows, the well becomes more triangular, mimicking the shape of the indium concentration profile. This change in the well shape also reduces the amount of polarization induced band bending. A reduction in the band bending acts similarly to a reduction in polarization and raises the GaN barriers across the device. This is most noticeable in the p-type region of Figure 16. 


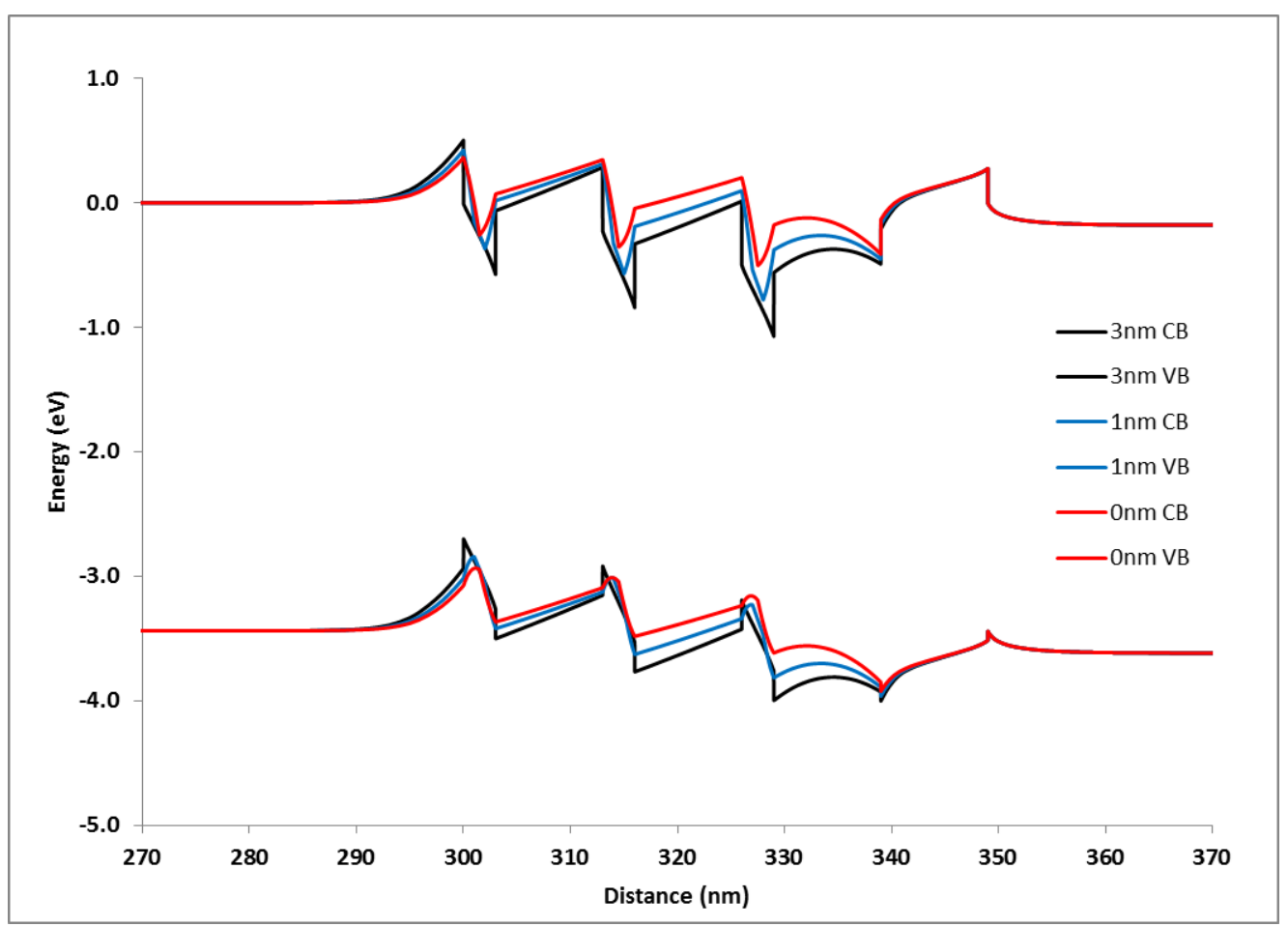

Figure 16: The band diagrams of the $\ln _{0.2} \mathrm{Ga}_{0.8} \mathrm{~N} 3 \mathrm{QW}$ heterostructure at a $3.5 \mathrm{~V}$ bias for trapezoidal indium profiles with base lengths of $3 \mathrm{~nm}$ (square), $1 \mathrm{~nm}$, and $0 \mathrm{~nm}$ (triangular). Narrowing the flat indium composition in the QW region reduces the polarization induced band bending, which thereby increases electron-hole overlap while raising the GaN barrier energy. The former leads to an increase in current density while the latter leads to a decrease. This creates similar, but not identical, effects to polarization reduction.

Just as with polarization reduction, a reduction in the indium flat band region causes both the CB and the VB to shift in energies. The GaN barrier energy band rises in both the $\mathrm{CB}$ and $\mathrm{VB}$, which causes a reduction in electron flow and an increase in hole flow. Since holes have poor mobility in nitrides, this facilitates the spreading of holes out of the p-type region of the device. Unlike the reduced polarization case, the slope of the GaN barriers are relatively unaffected and still impede both electron and hole flow through the LED device. The increase in the CB QW energies compared to the VB QW energies also leads to a blue shift in LED emission as the QWs get narrower. 


\subsubsection{Wavefunctions with Altered Quantum Well Shape}

A closer look at the middle QW in the LED device active region shows how QW shape dictates the electron and hole wavefunction. As the bottom (top for holes) of the QW narrows, the electron and hole wavefunctions get forced closer together. This increases the electron-hole overlap from $10.4 \%$ in the square $\mathrm{QW}$ to $26.6 \%$ and $32.9 \%$ in the trapezoidal and triangular $\mathrm{QWs}$, respectively. This increase in electron-hole overlap is the primary reason for increased current density at lower bias voltages.

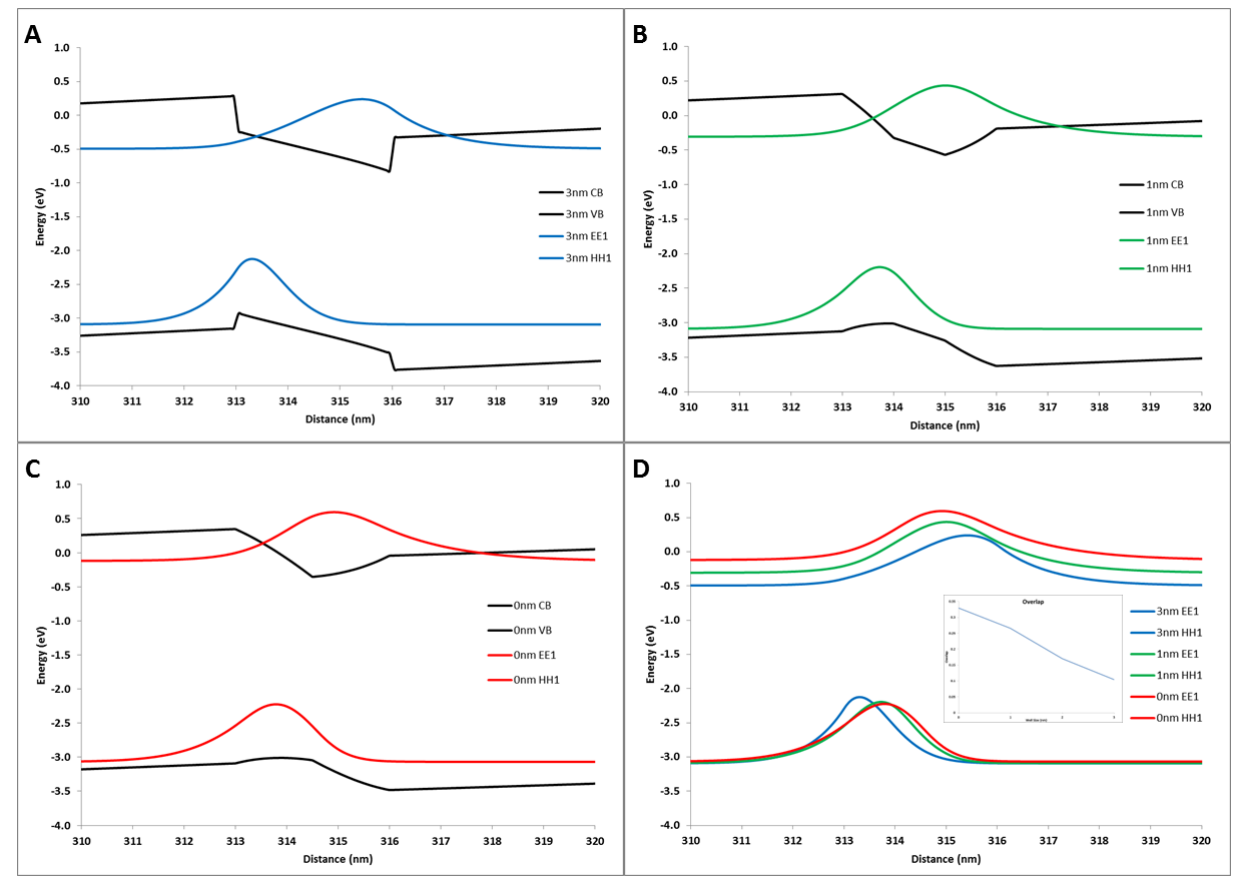

Figure 17: The electron-hole wavefunctions for the middle $Q W$ in the $\ln _{0.2} \mathrm{Ga}_{0.8} \mathrm{~N}$ $3 \mathrm{QW}$ heterostructure at a $3.5 \mathrm{~V}$ bias for trapezoidal indium profiles with base lengths of (A) $3 \mathrm{~nm}$ (overlap =10.4\%), (B) $1 \mathrm{~nm}$ (overlap $=26.6 \%$ ), and (C) $0 \mathrm{~nm}$ (overlap $=32.9 \%$ ). (D) This figure shows how the electron and hole wavefunctions move closer together as the flat indium region is reduced (inset graphically shows the trend with overlap). A definite increase in the electron energy is seen as overlap increases, creating a blueshift in the energy spectrum. 


\section{Discussion}

\subsection{Analysis of Polarization Reduction}

The reduction in polarization constants has a dramatic effect on the current density output as seen in Figure 12. From these results, it is clear why several papers reduce their simulated polarization effects to $40-60 \%$ of the theoretical values. ${ }^{15-19}$ This reduction in polarization constants is typically attributed to defect mediated charge shielding within the QW, however, experimental evidence for this assumption is rarely cited.

Both the $70 \%$ and $40 \%$ reduction in polarization constants led to an increased current density. This can be attributed to an increase in the electron-hole overlap integral as polarization decreases (Figure 14). An increase in the electron-hole overlap is directly correlated to an increased recombination rate as derived from Fermi's golden rule (Equation 47) and shown in Equation 59. This gives an expression for the current density that is a slight modification of Equation 99:

$$
J(\langle n \mid p\rangle)=q w\left(A n+B_{0}|\langle n \mid p\rangle|^{2} n^{2}+C n^{3}\right)
$$

where the radiative recombination coefficient $B$ is now proportional to the square of the overlap integral. From Equation 101, it is clear that the electron-hole overlap plays a dominant role in increasing the current density within the LED 3QW device.

The reduction in polarization plays a secondary role as it flattens out the sloping bands in Figure 13. The sloped bands in the QW and barrier regions generate an electric field, $\mathbf{E}=-\nabla \Phi$, that either accelerates or retards carrier flow. This is directly seen in Equations 62 and 63, where $\mathbf{J} \propto \mathbf{E}$. The relative slope of the band in Figure 13 then shows how it affects carrier flow, with a 
positive slope inducing current to the right and a negative slope inducing current to the left. The $3.5 \mathrm{~V}$ bias in Figure 13 produces a leftward moving current, which means that the GaN barrier regions of the MQW heterostructure are fighting the current flow at $100 \%$ polarization. As the polarization is reduced to $40 \%$, the GaN barrier's slope rotates to help more current flow while the $\mathrm{In}_{0.2} \mathrm{Ga}_{0.8} \mathrm{~N}$ QW becomes flatter.

\subsection{Analysis of Quantum Well Shape}

Narrowing the flat $\ln _{0.2} \mathrm{Ga}_{0.8} \mathrm{~N}$ region of the $\operatorname{InGaN} / \mathrm{GaN} 3 \mathrm{QW}$ heterostructure significantly improved the current density (Figure 15) similarly to that of polarization reduction (Figure 12). It accomplished this by increasing the net recombination rate of electron-hole pairs in the QW regions. Recent studies have also shown that trapezoidal indium profiles are desired in InGaN/GaN MQW device designs for their ability to increase the electron-hole overlap. ${ }^{28,29}$ The trapezoidal and triangular QWs are able to increase the electron-hole overlap by reducing the region of flat indium composition in the QW.

Abrupt boundary changes in the device heterostructure causes bound surface charges to form (Equation 20), which creates capacitive charge sheets. A graded indium composition would then reduce the strength of this change and spread it out over the distance of the grading as shown in Figure 16. This then guides the carriers into the center of the $\mathrm{QW}$ where they interact with the region of flat indium composition with a linearly sloped band due to the bound charges. This sloped band tends to pull the holes and electrons apart thereby reducing their overlap integral and their overall recombination rate. Narrowing this region of flat indium composition has the effect of pushing the electrons and holes together because they can no longer be pulled apart by the induced electric field of the bound charges. As the region of flat indium composition goes to zero, the bound charges go to zero as well. This creates regions of 
alternating polarization that now guides electrons and holes into the same point in the QW as opposed to the opposite sides of the QW. This effect significantly increases overlap as seen in Figure 17, although not as much as polarization reduction (Figure 14).

\subsection{Polarization vs. Well Shape}

A reduction in the polarization constants to $40 \%$ their original values significantly reduces the onset voltage of the $3 \mathrm{QW} \operatorname{In}_{0.2} \mathrm{Ga}_{0.8} \mathrm{~N}$ device and brings it closer to typical experimental values. ${ }^{26,27}$ Altering the InGaN well shape also has similar effects on the J-V characteristic of the InGaN/GaN MQW heterostructure. It is unclear whether this is the fault of the SiLENSe simulation software or the fault of the user.

Comparison of sections 3.1 and 3.2 clearly shows that altering the square shape of the InGaN QW in order to reduce the flat $\ln _{0.2} \mathrm{Ga}_{0.8} N$ region has similar effects as reducing the overall polarization constants of the LED MQW heterostructure. These similarities are closely tied to polarization's dominant role in allowing recombination of the electrons and holes. The fact that $\mathrm{QW}$ shape increases carrier recombination through similar mechanisms as polarization reduction warrants further investigation into the validity of the square $\mathrm{QW}$ model.

One possible explanation for the apparent reduction in polarization may come from the InGaN/GaN interfacial bound charge density spreading away from the interface. This can happen if the bound charges tunnel a few atoms away, creating a diffuse charge layer that spreads a little less than a nanometer from the surface of the interface. This creates an effect similar to the graded alloying of indium in the quantum well. A graded alloying profile of $1 \mathrm{~nm}$ smears out the polarization bound charges, thereby narrowing the well and increasing electron-hole overlap (section 3.2). Therefore, tunneling of bound charges 
accounts for some polarization reduction in InGaN QW devices without altering the indium profile.

The use of square $\mathrm{QW}$ band diagrams to simulate experimental devices may not be sufficient for complex devices. It has already been shown that a single QW GaN/InGaN/AIGaN LED with a nominal square well indium composition actually obtains a skewed Gaussian composition due to thermodynamic effects. ${ }^{4,25}$ Therefore, the discrepancy between simulation and experiment may be partially due to the inaccuracies of the simulation models employed by the user, and further investigation into modeling realistic indium profiles is needed.

\section{Conclusion}

This project involved the understanding and application of current semiconductor physical models to simulate the $\mathrm{J}-\mathrm{V}$ characteristics of a $3 \mathrm{QW}$ InGaN/GaN heterostructure LED with an AIGaN EBL. The device was simulated with different polarization constants to mimic the effect polarization shielding and different InGaN QW shapes mimic the effect of a non-square indium composition. The values of the polarization constants were reduced from their original values (Table I) to $70 \%$ and $40 \%$. The different InGaN QW shapes simulated had a $3 \mathrm{~nm}$ base with a peak indium concentration width of 3 $\mathrm{nm}$ (square), $1 \mathrm{~nm}$, and $0 \mathrm{~nm}$ (triangle). The reduction in polarization constants led to a dramatic shift in the $\mathrm{J}-\mathrm{V}$ characteristic from an onset voltage of $4.0 \mathrm{~V}$ at $100 \%$ polarization to $3.4 \mathrm{~V}$ at $70 \%$ polarization and $3.0 \mathrm{~V}$ at $40 \%$ polarization. These values are much closer to the experimental onset voltages, typically around $3.0 \mathrm{~V}$, and can be attributed to an increase in electron-hole overlap as well as a decrease in polarization induced band bending. The electron-hole overlap increased from $10.4 \%$ for the $100 \%$ polarization to 
$25.0 \%$ for $70 \%$ polarization and $52.7 \%$ for $40 \%$ polarization. Altering the QW shape also had similar effects as polarization reduction with a shift in the J-V characteristic. The onset voltage for the $3 \mathrm{~nm} \mathrm{QW}$ was $4.0 \mathrm{~V}$ and decreased to 3.8 $\mathrm{V}$ for the $1 \mathrm{~nm}$ trapezoidal $\mathrm{QW}$ and $3.5 \mathrm{~V}$ for the $0 \mathrm{~nm}$ triangular $\mathrm{QW}$. This can also be attributed to an increase in the electron-hole overlap from $10.4 \%$ for the $3 \mathrm{~nm}$ QW to $26.6 \%$ for the $1 \mathrm{~nm}$ trapezoidal QW and $32.9 \%$ for the $0 \mathrm{~nm}$ triangular QW. These results indicate that both changes in effective polarization and changes in QW shape can lead to increased current densities and carrier recombination rates through an increased electron-hole overlap. This indicates that a realistic $\mathrm{QW}$ profile plays a dominant role in the current characteristics of a nitride based heterostructure LED. 


\section{References}

[1] Department of Energy. Why SSL. <http:

//www1. eere. energy.gov/buildings/ssl/sslbasics_whyssl.html>, March 2012.

[2] Navigant Consulting, Inc. Energy savings potential of solid-state lighting in general illumination applications. Technical report, Department of Energy, 2012.

[3] Isamu Akasaki. Key inventions in the history of nitride-based blue LED and LD. Journal of Crystal Growth, 300:2-10, 2007.

[4] Christian Kisielowski, Zuzanna Liliental-Weber, and Shuji Nakamura. Atomic scale indium distribution in a $\mathrm{GaN} / \mathrm{In}_{0.43} \mathrm{Ga}_{0.57} \mathrm{~N} / \mathrm{Al}_{0.1} \mathrm{Ga}_{0.9} \mathrm{~N}$ quantum well structure. Jpn. J. Appl. Phys., 36, 1997.

[5] A. E. Romanov, T. J. Baker, S. Nakamura, and J.S. Speck. Strain-induced polarization in wurtzite III-nitride semipolar layers. Journal of Applied Physics, 100, 2006.

[6] Egon E. Loebner. Subhistories of the light emitting diode. IEEE Transactions on Electron Devices, ED-23(7), 1976.

[7] Nikolay Zheludev. The life and times of the led - a 100-year history. Nature Photonics, 1, 2007.

[8] Felix Bloch. Uber die quantenmechanik der elektronen in kristallgittern. Zeits. f. Physik, 52:555, 1928.

[9] W. Shockley. Theory of p-n junctions. Bell System Technical Journal, 28, 1949.

[10] K. Lehovec. New photoelectric devices utilizing carrier injection. Proc. IRE, pages 1407-1409, 1952. 
[11] H. P. Maruska. A brief history of GaN blue light-emitting diodes. Office of Naval Research, National Compound Semiconductor Roadmap.

[12] J. J. Tietjen H. P. Maruska. Applied Physics Letters, 15:327, 1969.

[13] M. Senoh N. Iwasa S. Nakamura, T. Mukai. Japan Journal of Applied Physics, 31:L970, 1992.

[14] Daniel F. Feezell, Mathew C. Schmidt, Steven P. DenBaars, and Shuji Nakamura. Development of nonpolar and semipolar InGaN/GaN visible light-emitting diodes. MRS Bulletin, 34, May 2009.

[15] Ü. Özgür, X. Ni, J. Lee, S. Liu, S. Okur, V. Avrutin, A. Matulionis, and H. Morkoç. Ballistic transport in ingan-based leds: impact on efficiency. Semiconductor Science and Technology, 26, 2011.

[16] Y.-K. Kuo, J.-Y. Chang, and M.-C. Tsai. Enhancement in hole-injection efficiency of blue InGaN light-emitting diodes from reduced polarization by some specific designs for the electron blocking layer. Optics Letters, 35(19), 2010.

[17] Tsun-Hsin Wang, Jih-Yuan Chang, Miao-Chan Tsai, and Yen-Kuang Kuo. Efficiency enhancement of blue InGaN leds with indium composition graded InGaN barriers. Proc. of SPIE, 7954, 2011.

[18] C. H. Wang, C. C. Ke, C. Y. Lee, S. P. Chang, W. T. Chang, J. C. Li, Z. Y. Li, H. C. Yang, H. C. Kuo, T. C. Lu, and S. C. Wang. Hole injection and efficiency droop improvement in InGaN/GaN light-emitting diodes by band-engineered electron blocking layer. Applied Physics Letters, 97, 2010.

[19] C. H. Wang, S. P. Chang, P. H. Ku, J. C. Li, Y. P. Lan, C. C. Lin, H. C. Yang, H. C. Kuo, T. C. Lu, S. C. Wang, and C. Y. Chang. Hole transport improvement in InGaN/GaN light-emitting diodes by graded-composition multiple quantum barriers. Applied Physics Letters, 99, 2011. 
[20] STR, Inc. Physical Model. http:

//www1. eere. energy .gov/buildings/ssl/sslbasics_whyssl.html, 2009.

[21] David J. Griffiths. Indroduction to Electrodynamics. Prentice Hall, Inc., 1999.

[22] Shun Lien Chuang. Physics of Optoelectronic Devices. John Wiley \& Sons, Inc., 1995.

[23] David J. Griffiths. Introduction to Quantum Mechanics. Pearson Education, Inc., 2005.

[24] STR. SiLENSe Physics Summary, version 5.2 edition, 2011.

[25] T. P. Bartel and C. Kisielowski. A quantitative procedure to probe for compositional inhomogeneities in $\ln _{x} \mathrm{Ga}_{1-x} \mathrm{~N}$ alloys. Ultramicroscopy, 108:1420-1426, 2008.

[26] Wonseok Lee, Min-Ho Kim, Di Zhu, Ahmed N. Noemaun, Jong Kyu Kim, and E. F. Schubert. Growth and characteristics of GalnN/GalnN multiple quantum well light-emitting diodes. Journal of Applied Physics, 107:63102-6, 2010.

[27] S.-H. Han, D.-Y. Lee, S.-J. Lee, C.-Y. Cho, M.-K. Kwon, S. P. Lee, D. Y. Noh, D.-J. Kim, Y. C. Kim, and S.-J. Park. Effect of electron blocking layer on efficiency droop in InGaN/GaN multiple quantum well light-emitting diodes. Applied Physics Letters, 94, 2009.

[28] Sang-Heon Han, Dong-Yul Lee, Hyun-Wook Shim, Gwon-Chul Kim, Young Sun Kim, Sung-Tae Kim, Sang-Jun Lee, Chu-Young Cho, and Seong-Ju Park. Improvement of efficiency droop in InGaN/GaN multiple quantum well light-emitting diodes with trapezoidal wells. J. Phys. D: Appl. Phys., 43, 2010. 
[29] Ray-Ming Lin, Mu-Jen Lai, Liann-Be Chang, Chou-Hsiung Huang, and Chang-Ho Chen. Effect of trapezoidal-shaped well on efficiency droop in InGaN-based double-heterostructure light-emitting diodes. International Journal of Photoenergy, 2012, 2011. 